Submitted to the Annals of Statistics

Supplementary document to

\title{
LOCAL AND GLOBAL ASYMPTOTIC INFERENCE IN SMOOTHING SPLINE MODELS
}

\author{
By Zuofeng Shang ${ }^{\ddagger}$ And Guang Cheng ${ }^{\S}$ \\ University of Notre Dame and Purdue University \\ August 27, 2013
}

In this document, we first give a table that lists the notation of the paper, and then give the proofs. In particular, we describe and prove the minimax results for the PLRT.

We organize this document as follows:

- In Section S.1, we give a table defining our notation, and indicating the page numbers of the first occurences.

- Sections S.2, S.3, and S.4 include the proofs of Proposition 2.1, Proposition 2.2, and Lemma 3.1.

- In Section S.5, we prove the concentration inequality in Lemma 3.2.

- In Section S.6, we prove Proposition 3.3, i.e., the convergence rate of $\widehat{g}_{n, \lambda}$.

- In Section S.7, we prove Theorem 3.4, i.e., the FBR. In Section S.8, we prove the pointwise asymptotic normality of the smoothing spline estimate in Theorem 3.5.

- In Section S.9, we prove Corollary 3.7 on the pointwise asymptotic normality of $\widehat{g}_{n, \lambda}$ in $L_{2}$ regression.

- In Section S.10, we sketch the proof of another technical tool, i.e., the restricted FBR in Theorem 4.3, which is used to establish the asymptotic null distribution of the local LRT.

- In Section S.11, we show the Wilks phenomenon of the local LRT.

- In Section S.12, we prove Corollary 4.5.

- In Section S.13, we demonstrate the validity of the proposed SCB.

- In Section S.14, we prove Proposition 5.2, i.e., the equivalent kernel conditions.

- In Section S.15, we derive the null limiting distribution of the PLRT.

- In Section S.16, we prove Theorem 5.4, i.e., when the data are normal, the PLRT attains the optimal minimax rates of testing. We further extend this result to a more general modeling

\footnotetext{
${ }^{\ddagger}$ Postdoctoral Fellow.

${ }^{\S}$ Corresponding Author. Associate Professor. Research Sponsored by NSF, DMS-0906497, and CAREER Award DMS-1151692
} 
framework in Section S.17.

- In Section S.18, we show Lemma 6.1.

- We give two figures for Example 6.1 showing the performance of the proposed ACI and SCB.

The reference labels of the equations, theorems, propositions, and lemmas in this document are consistent with those in the main text of the paper. 


\begin{tabular}{|c|c|c|}
\hline Notation & Meaning & First appearing in page No. \\
\hline $\bar{Y}$ & response & 4 \\
\hline$Z$ & covariate & 4 \\
\hline$T$ & $T=(Y, Z)$ denotes the full data variable & 4 \\
\hline $\mathcal{Y}$ & range of $Y$ & 4 \\
\hline $\mathbb{I}$ & $\mathbb{I}=[0,1]$ denotes the range of $Z$ & 4 \\
\hline$F$ & the link function & 4 \\
\hline$g_{0}$ & the true function & 4 \\
\hline$Q(y ; \mu)$ & the quasi-likelihood function & 4 \\
\hline$\ell(y ; a)$ & the general criteria function & 4 \\
\hline$m$ & the roughness degree of $g_{0}$ & 4 \\
\hline$J(\cdot, \cdot)$ & the penalty function & 4 \\
\hline $\mathcal{H}$ & the parameter space for $g$ & 4 \\
\hline$H^{m}(\mathbb{I})$ & the Sobolev space on $[0,1]$ & 4 \\
\hline$H_{0}^{m}(\mathbb{I})$ & the homogeneous Sobolev space on $[0,1]$ & 4 \\
\hline$\ell_{n, \lambda}$ & the penalized empirical criteria function & 4 \\
\hline$\widehat{g}_{n, \lambda}$ & the unconstrained estimate of $g$ & 4 \\
\hline$\lambda$ & the smoothing parameter & 4 \\
\hline $\mathcal{N}_{m}$ & the null space for $J$ & 4 \\
\hline $\mathcal{I}_{0}$ & the range of $g_{0}$ & 4 \\
\hline $\mathcal{I}$ & a bounded open interval including $\mathcal{I}_{0}$ & 4 \\
\hline$\dot{\ell}_{a}, \ddot{\ell}_{a}, \ell_{a}^{\prime \prime \prime}$ & the first-, second- and third-order partial derivatives w.r.t. $a$ & 4 \\
\hline$I(Z)$ & $-E\left\{\ddot{\ell}_{a}\left(Y ; g_{0}(Z)\right) \mid Z\right\}$ & 5 \\
\hline$\langle\cdot, \cdot\rangle,\|\cdot\|$ & the inner product and norm & 5 \\
\hline$K$ & the reproducing kernel function & 6 \\
\hline$K_{z}$ & the function determined by $K_{z}(\cdot)=K(z, \cdot)$ & 6 \\
\hline$V$ & $V(g, \widetilde{g})=E\{I(Z) g(Z) \widetilde{g}(Z)\}$ & 6 \\
\hline$W_{\lambda}$ & the penalty operator & 6 \\
\hline$i \hat{d}$ & the identity operator & 6 \\
\hline$a_{\nu} \asymp b_{\nu}$ & asymptotically equivalent & 6 \\
\hline$a_{\nu} \sim b_{\nu}$ & asymptotically equal & 6 \\
\hline$\|\cdot\|_{\text {sup }}$ & the supremum norm & 6 \\
\hline$\gamma_{\nu}$ & the eigenvalue & 6 \\
\hline$h_{\nu}$ & the eigenfunction function corresponding to $\gamma_{\nu}$ & 6 \\
\hline$D$ & the Fréchet differential operator & 7 \\
\hline$S_{n, \lambda}$ & $=D \ell_{n, \lambda}$ & 7 \\
\hline$h$ & $=\lambda^{1 /(2 m)}$ & 7 \\
\hline$\|\cdot\|_{\mathcal{H}}$ & a norm stronger than $\|\cdot\|$ & 8 \\
\hline$\lambda^{*}$ & a sequence satisfying $\lambda^{*} \asymp n^{-2 m /(2 m+1)}$ & 8 \\
\hline$h^{*}$ & $=\left(\lambda^{*}\right)^{1 /(2 m)}$ & 8 \\
\hline$g_{0}^{*}$ & $=\left(i d-W_{\lambda}\right) g_{0}$ & 9 \\
\hline$\sigma_{z_{0}}^{2}$ & the asymptotic variance of $\widehat{g}_{n, \lambda}\left(z_{0}\right)$ & 9 \\
\hline$b_{z_{0}}$ & the asymptotic estimation bias of $\widehat{g}_{n, \lambda}\left(z_{0}\right)$ & 9 \\
\hline$h^{\dagger}$ & $=h \sigma^{1 / m}$ & 11 \\
\hline$I_{1}, I_{2}$ & two explicit integrals & 11 \\
\hline$a\left(h^{\dagger}\right)$ & a quantity introduced by Wahba (1983) & 12 \\
\hline$\widehat{g}_{n, \lambda}^{0}$ & the optimizer constrained with $g\left(z_{0}\right)=0$ & 13 \\
\hline $\mathcal{H}_{0}$ & a subspace of $\mathcal{H}$ with restriction $g\left(z_{0}\right)=0$ & 13 \\
\hline$K^{*}$ & a restricted version of $K$ in $\mathcal{H}_{0}$ & 13 \\
\hline$W_{\lambda}^{*}$ & a restricted version of $W_{\lambda}$ in $\mathcal{H}_{0}$ & 13 \\
\hline$r_{n}$ & rate of convergence & 14 \\
\hline$c_{0}$ & a scaling constant in local LRT & 14 \\
\hline$\omega(\cdot)$ & an equivalent kernel function & 15 \\
\hline$\omega_{0}$ & an equivalent kernel function in the specific $L_{2}$ regression & 17 \\
\hline$r_{K}, \rho_{K}, \sigma_{K}^{2}, u_{n}$ & constants in the PLRT & 19 \\
\hline$\widehat{\sigma}^{2}$ & estimated variance & 22 \\
\hline
\end{tabular}

TABLE 5

A table that lists all useful notation, their meanings and where they first appear.

S.1. Notation Table. 
S.2. Proof of Proposition 2.1. Based on the definition (2.8), we can write $\|g\|^{2}=V(g, g)+$ $\lambda J(g, g)$, and then plug in the Fourier expansion of $g$ to obtain the explicit expression of $\|g\|^{2}$. A direct calculation reveals that

$$
\left\langle g, h_{\nu}\right\rangle=\left\langle\sum_{\mu} V\left(g, h_{\mu}\right) h_{\mu}, h_{\nu}\right\rangle=V\left(g, h_{\nu}\right)\left(1+\lambda \gamma_{\nu}\right)
$$

for any $g \in \mathcal{H}$ and $\nu \in \mathbb{N}$. It follows by (S.1) that $V\left(K_{z}, h_{\nu}\right)=\left\langle K_{z}, h_{\nu}\right\rangle /\left(1+\lambda \gamma_{\nu}\right)=h_{\nu}(z) /\left(1+\lambda \gamma_{\nu}\right)$. Hence, we can obtain the expression of $K_{z}(\cdot)$ by using $K_{z}(\cdot)=\sum_{\nu} V\left(K_{z}, h_{\nu}\right) h_{\nu}(\cdot)$. Furthermore, (S.1) implies that $V\left(W_{\lambda} h_{\nu}, h_{\mu}\right)=\left\langle W_{\lambda} h_{\nu}, h_{\mu}\right\rangle /\left(1+\lambda \gamma_{\mu}\right)=\lambda \gamma_{\mu} \delta_{\mu \nu} /\left(1+\lambda \gamma_{\mu}\right)$, for any $\nu, \mu \in \mathbb{N}$. Finally, we can conclude the proof of Proposition 2.1 by using $W_{\lambda} h_{\nu}(\cdot)=\sum_{\mu} V\left(W_{\lambda} h_{\nu}, h_{\mu}\right) h_{\mu}$.

S.3. Proof of Proposition 2.2. The usual $L_{2}$-inner product is defined to be $\langle g, \xi\rangle_{L_{2}}=\int_{0}^{1} g(z) \xi(z) d z$. Let $D$ be the differential operator, i.e., $D \phi=\frac{d}{d z} \phi$, and $\omega=1 /(I \pi)$. Thus, $\omega \in C^{m}(\mathbb{I})$ is positive and finitely upper bounded. It follows from [5] that the growing rate for $\gamma_{\nu}$ is of order $\nu^{2 m}$. Since the operator $L_{0}=(-1)^{m} \omega D^{2 m}$ is self-adjoint under the inner product $V$, that is, $V\left(L_{0} g, \xi\right)=V\left(g, L_{0} \xi\right)$ for any $\xi, g \in C^{2 m}(\mathbb{I})$ satisfying the boundary conditions in (2.11), orthogonality and completeness of $h_{\nu}$ under $V$ thus follow from Theorem 2.1 (pp. 189) and Theorem 4.2 (pp. 199) of [8] with the usual $L_{2}$-inner product $\langle,\rangle_{L_{2}}$ replaced with $V$. Therefore, when $h_{\nu}$ are normalized to $V\left(h_{\nu}, h_{\nu}\right)=1$, they form an orthonormal and complete set in $L_{2}(\mathbb{I} ; V)$.

Next we show that $h_{\nu}^{(m)}, \nu \geq m$, are complete in $L_{2}(\mathbb{I})$ under $\langle,\rangle_{L_{2}}$. The idea follows from arguments on page 147 of [31]. The eigenspace corresponding to the zero eigenvalue contains functions $\phi$ that satisfy $(-1)^{m} \phi^{(2 m)}=0$ with boundary conditions $\phi^{(j)}(0)=\phi^{(j)}(1)=0$ for $j=m, \ldots, 2 m-1$, thus it follows from [49] that this eigenspace is $\mathcal{P}_{m-1}$, i.e., the set of all polynomials of degree at most $m-1$. Let $h_{\nu}, \nu=0, \ldots, m-1$, be the orthonormal basis (under $V$ ) of $\mathcal{P}_{m-1}$ corresponding to $\gamma_{0}=\ldots=\gamma_{m-1}=0$. Note $\gamma_{\nu}>0$ for $\nu \geq m$. For $g \in L_{2}(\mathbb{I})$ such that for any $\nu \geq m, \int_{0}^{1} g h_{\nu}^{(m)}=0$, let $\xi$ be a solution of $\xi^{(m)}=g$, then using integration by parts we have $0=\int_{0}^{1} \xi h_{\nu}^{(2 m)}=(-1)^{m} \gamma_{\nu} V\left(\xi, h_{\nu}\right)$. Therefore $V\left(\xi, h_{\nu}\right)=0$ for any $\nu \geq m$. By completeness of $h_{\nu}, \xi$ must be a linear combination of $h_{0}, \ldots, h_{m-1}$, a polynomial with degree at most $m-1$. So $g=\xi^{(m)}=0$ implying the completeness of $h_{\nu}^{(m)} / \gamma_{\nu}^{1 / 2}$ for $\nu \geq m$ in $L_{2}(\mathbb{I})$ under $\langle,\rangle_{L_{2}}$. Now, for any $\tilde{g} \in \mathcal{H}$, by completeness of $h_{\nu}$ in $L_{2}(\mathbb{I})$ under $V$-norm, $\tilde{g}=\sum_{\nu \in \mathbb{N}} V\left(\tilde{g}, h_{\nu}\right) h_{\nu}$ with convergence in $V$-norm. Since $V\left(\tilde{g}, h_{\nu}\right)=\int_{0}^{1} \tilde{g}^{(m)} h_{\nu}^{(m)} / \gamma_{\nu}$, by completeness of $h_{\nu}^{(m)} / \gamma_{\nu}^{1 / 2}$ for $\nu \geq m$ in $L_{2}(\mathbb{I})$ in usual $\|\cdot\|_{L_{2}}$-norm, $\tilde{g}^{(m)}=\sum_{\nu \geq m}\left\langle\tilde{g}^{(m)}, h_{\nu}^{(m)}\right\rangle_{L_{2}} h_{\nu}^{(m)} / \gamma_{\nu}=\sum_{\nu \geq m} V\left(\tilde{g}, h_{\nu}\right) h_{\nu}^{(m)}$ with convergence in usual $L_{2}$-norm. This implies $\tilde{g}=\sum_{\nu} V\left(\tilde{g}, h_{\nu}\right) h_{\nu}$ converges in $\|\cdot\|$.

Next we show the uniform boundedness of $h_{\nu}$. We only consider those $h_{\nu}$ corresponding to nonzero $\gamma_{\nu}$. If $\gamma_{\nu} \neq 0$ and $h_{\nu}$ satisfy $(-1)^{m} h_{\nu}^{(2 m)}=\gamma_{\nu} I \pi h_{\nu}$ and $V\left(h_{\nu}, h_{\nu}\right)=1$, then using the boundary conditions in (2.11) and integration by parts, one can check that $J\left(h_{\nu}, h_{\nu}\right)=\gamma_{\nu}$. Dividing $I \pi$ and taking $m$-order derivatives on both sides, one obtains $L h_{\nu}^{(m)}=\gamma_{\nu} h_{\nu}^{(m)}$ with 
$h_{\nu}^{(m+j)}(0)=h_{\nu}^{(m+j)}(1)=0, j=0, \ldots, m-1$, where $L=(-1)^{m} \sum_{j=0}^{m}\left(\begin{array}{c}m \\ j\end{array}\right) \omega^{(j)} D^{2 m-j}$. Therefore, $h_{\nu}^{(m)}$ is an eigenfunction of $L$ with eigenvalue $\gamma_{\nu}$. Denote the eigenfunctions and eigenvalues of $L$ to be $\psi_{\nu}$ and $\lambda_{\nu}$ subject to $\psi_{\nu}^{(j)}(0)=\psi_{\nu}^{(j)}(1)=0, j=0, \ldots, m-1$. We need to transform $L$ to normal form. Let $t(z)=\int_{0}^{z}[I(s) \pi(s)]^{1 /(2 m)} d s / C$ and $C=\int_{0}^{1}[I(z) \pi(z)]^{1 /(2 m)} d z$. Define $\phi_{\nu}(t(z))=\psi_{\nu}(z)$. Then by a direct examination, $\phi_{\nu}$ satisfies the following differential equation

$\phi_{\nu}^{(2 m)}(t)+q_{2 m-1}(t) \phi_{\nu}^{(2 m-1)}(t)+\ldots+q_{0}(t) \phi_{\nu}(t)=\rho_{\nu} \phi_{\nu}(t), \phi_{\nu}^{(j)}(0)=\phi_{\nu}^{(j)}(1)=0, j=0, \ldots, m-1$

where $q_{j}, j=0, \ldots, 2 m-1$, are coefficient functions depending only on $I \pi$ and $m$, and $\rho_{\nu}=\lambda_{\nu} C^{2 m}$. In general the form of $q_{j}$ are complicated though they can be determined by Faá di Bruno's formula ([25]). As an illustration, when $m=2, q_{0}(t)=0, q_{3}(t)=-(C / 4) \omega^{(1)}(z(t)) \omega(z(t))^{-3 / 4}$, $q_{2}(t)=-\left(C^{2} / 4\right)\left(\omega^{(1)}(z(t))\right)^{2} \omega(z(t))^{-3 / 2}$, and

$q_{1}(t)=C^{3}\left(-5 \omega(z(t))^{-9 / 4}\left(\omega^{(1)}(z(t))\right)^{3} / 64+3 \omega(z(t))^{-5 / 4} \omega^{(1)}(z(t)) \omega^{(2)}(z(t)) / 16-\omega(z(t))^{-1 / 4} \omega^{(3)}(z(t))\right)$,

where $z(t)$ is the inverse function of $t(z)$ and $b_{2}(z)=[I(z) \pi(z)]^{1 / 4}$. Define

$$
u_{\nu}(t)=\phi_{\nu}(t) \exp \left(\frac{1}{2 m} \int_{0}^{t} q_{2 m-1}(s) d s\right)
$$

then (S.2) is equivalent to

$$
\tilde{L} u_{\nu} \equiv u_{\nu}^{(2 m)}(t)+p_{2 m-2}(t) u_{\nu}^{(2 m-2)}+\ldots+p_{0}(t) u_{\nu}(t)=\rho_{\nu} u_{\nu}(t)
$$

with the boundary conditions $u_{\nu}^{(j)}(0)=u_{\nu}^{(j)}(1)=0, j=0, \ldots, m-1$. Note (S.4) is the classic form of differential systems discussed in [5]. According to [5], $\rho_{\nu}$ are simple due to the regular boundary conditions, and the residual of the Green function $G\left(z_{1}, z_{2} ; \rho\right)$ for $\tilde{L}-\rho I$ at pole $\rho_{\nu}$ is given by $\frac{u_{\nu}\left(t_{1}\right) u_{\nu}\left(t_{2}\right)}{\left\|u_{\nu}\right\|_{L_{2}}^{2}}$, where $\|\cdot\|_{L_{2}}$ denotes the usual $L_{2}$-norm. On the other hand, the residue can also be represented by $\frac{1}{2 \pi \sqrt{-1}} \int_{\Gamma_{\rho_{\nu}}} 2 m \zeta^{2 m-1} G\left(t_{1}, t_{2}, \zeta^{2 m}\right) d \zeta$ (pp. 722, [45]), where $\zeta=\rho^{1 /(2 m)}, \Gamma_{\rho_{\nu}}$ denotes the contour centered around pole $\rho_{\nu}$ with suitably small radius. By equation (56) and the discussions below in [5], $2 m \zeta^{2 m-1} G\left(t_{1}, t_{2} ; \zeta^{2 m}\right)$ is uniformly bounded for $t_{1}, t_{2} \in \mathbb{I}$, thus the residue is uniformly bounded for all $t_{1}, t_{2}$. In particular, letting $t_{1}=t_{2}=t$, we get $\left|u_{\nu}(t)\right| \leq c\left\|u_{\nu}\right\|_{L_{2}}$ for any $t \in \mathbb{I}$ with a universal constant $c>0$. Since $q_{2 m-1}$ achieves finite upper and lower bounds on $\mathbb{I}$, by (S.3), there is a universal constant $c_{1}>0$ such that for any $\nu,\left\|\phi_{\nu}\right\|_{\text {sup }} \leq c_{1}\left\|\phi_{\nu}\right\|_{L_{2}}$. Now, using $\phi_{\nu}(t(z))=\psi_{\nu}(z)$, we get $\left\|\psi_{\nu}\right\|_{\text {sup }}^{2}=\left\|\phi_{\nu}\right\|_{\text {sup }}^{2} \leq c_{1}^{2}\left\|\phi_{\nu}\right\|_{L_{2}}^{2}=c_{1}^{2} \int_{0}^{1}\left|\phi_{\nu}(t)\right|^{2} d t=c_{1}^{2} \int_{0}^{1}\left|\phi_{\nu}(t(z))\right|^{2}|I(z) \pi(z)|^{1 /(2 m)} d z \leq c_{1}^{2} c_{I \pi}^{2}\left\|\psi_{\nu}\right\|_{L_{2}}^{2}$,

where $c_{I \pi}$ is a constant depending only on $I \pi$ and $m$. So $\left\|\psi_{\nu}\right\|_{\text {sup }} \leq c_{1} c_{I \pi}\left\|\psi_{\nu}\right\|_{L_{2}}$. Letting $\psi_{\nu}=h_{\nu}^{(m)}$ and using the fact that $\left\|h_{\nu}^{(m)}\right\|_{L_{2}}^{2}=\gamma_{\nu}$, we have $\left\|h_{\nu}^{(m)}\right\|_{\text {sup }} \leq c_{1} c_{I \pi} \gamma_{\nu}^{1 / 2}$, for any $\nu \in \mathbb{N}$. 
By the Sobolev embedding theorem $([1]),\left\|h_{\nu}\right\|_{\text {sup }}^{2} \leq c^{2}\left(V\left(h_{\nu}, h_{\nu}\right)+J\left(h_{\nu}, h_{\nu}\right)\right)=c^{2}\left(1+\gamma_{\nu}\right)$. By Theorem 5 of [49], for any $j=1, \ldots, m$, there is a constant $C_{j}>0$ such that $\left\|h_{\nu}^{(j)}\right\|_{\text {sup }} \leq C_{j}(1+$ $\left.\gamma_{\nu}\right)^{1 / 2}, \forall \nu \in \mathbb{N}$. Therefore, taking the $m$ th-order derivative on both sides of $(-1)^{m} h_{\nu}^{(2 m)}=\gamma_{\nu} I \pi h_{\nu}$, one can show that $\left\|h_{\nu}^{(3 m)}\right\|_{\text {sup }} \leq \gamma_{\nu} \sum_{j=0}^{m}\left(\begin{array}{c}m \\ j\end{array}\right)\left\|(I \pi)^{(m-j)}\right\|_{\text {sup }} \cdot\left\|h_{\nu}^{(j)}\right\|_{\text {sup }} \leq c_{2}\left(1+\gamma_{\nu}\right)^{3 / 2}$ for some constant $c_{2}>0$ and any $\nu$. Again, by Theorem 5 of [49] for $h_{\nu}^{(m)}$ and $\epsilon=\gamma_{\nu}^{-1 /(2 m)}$, we have $\left\|h_{\nu}^{(2 m)}\right\|_{\text {sup }} \leq C_{m}^{\prime}\left(1+\gamma_{\nu}\right)$, which implies $\left\|h_{\nu}\right\|_{\text {sup }} \leq C_{m}^{\prime}\left(\inf _{z}|I(z)|\right)^{-1}\left(1+\gamma_{\nu}\right) / \gamma_{\nu} \leq C_{m}^{\prime \prime}$, with a universal constant $C_{m}^{\prime \prime}$ unrelated to $\nu$. This proves the desired uniform boundedness of $h_{\nu}$.

S.4. Proof of Lemma 3.1. For any $z \in \mathbb{I},\left|\left\langle K_{z}, g\right\rangle\right| \leq\left\|K_{z}\right\| \cdot\|g\|$. So, we only need to find the upper bound for $\left\|K_{z}\right\|$. By Proposition 2.1 and the boundedness of $h_{\mu}$, we have

$$
\left\|K_{z}\right\|^{2}=K(z, z)=\sum_{\mu \in \mathbb{Z}} \frac{\left|h_{\mu}(z)\right|^{2}}{1+\lambda \gamma_{\mu}} \leq C \sum_{\mu \in \mathbb{Z}} \frac{1}{1+\lambda \gamma_{\mu}} \leq c_{m}^{2} \lambda^{-1 /(2 m)}=c_{m}^{2} h^{-1}
$$

where $c_{m}>0$ is a constant that does not rely on $z$ and $h$. So $\left\|K_{z}\right\| \leq c_{m} h^{-1 / 2}$.

S.5. Proof of Lemma 3.2. For any $g, f \in \mathcal{G}$, by Lemma 3.1,

$$
\begin{aligned}
\left\|\left(\psi_{n}(T ; f)-\psi_{n}(T ; g)\right) K_{Z}\right\| & \leq c_{m}^{-1} h^{1 / 2}\|f-g\|_{\text {sup }} \cdot\left\|K_{Z}\right\| \\
& \leq c_{m}^{-1} h^{1 / 2}\|f-g\|_{\text {sup }} \cdot c_{m} h^{-1 / 2}=\|f-g\|_{\text {sup }} .
\end{aligned}
$$

By Theorem 3.5 of [38], for any $t>0, P\left(\left\|Z_{n}(f)-Z_{n}(g)\right\| \geq t\right) \leq 2 \exp \left(-\frac{t^{2}}{8\|f-g\|_{\text {sup }}^{2}}\right)$. Then by Lemma 8.1 in [27], we have \|\|$Z_{n}(g)-Z_{n}(f)\|\|_{\psi_{2}} \leq 8\|g-f\|_{\text {sup }}$, where $\|\cdot\|_{\psi_{2}}$ denotes the Orlicz norm associated with $\psi_{2}(s) \equiv \exp \left(s^{2}\right)-1$. It follows by Theorem 8.4 of [27] that for arbitrary $\delta>0$,

$\begin{aligned}\left\|\sup _{\substack{g, f \in \mathcal{G} \\\|g-f\|_{\text {sup }} \leq \delta}}\right\| Z_{n}(g)-Z_{n}(f)\|\|_{\psi_{2}} & \leq C^{\prime}\left(\int_{0}^{\delta} \sqrt{\log \left(1+N\left(\delta, \mathcal{G},\|\cdot\|_{\text {sup }}\right)\right)}+\delta \sqrt{\log \left(1+N\left(\delta, \mathcal{G},\|\cdot\|_{\text {sup }}\right)^{2}\right)}\right) \\ & \asymp h^{-(2 m-1) /(4 m)} \delta^{1-1 /(2 m)} .\end{aligned}$

So, again, by Lemma 8.1 in [27],

$$
P\left(\sup _{\substack{g \in \mathcal{G} \\\|g\|_{\text {sup }} \leq \delta}}\left\|Z_{n}(g)\right\| \geq t\right) \leq 2 \exp \left(-h^{(2 m-1) /(2 m)} \delta^{-2+1 / m} t^{2}\right) .
$$

Let $b_{n}=n^{1 / 2} h^{-(2 m-1) /(4 m)}, \varepsilon=b_{n}^{-1}, \gamma=1-1 /(2 m), T_{n}=(5 \log \log n)^{1 / 2}$, and $Q_{\varepsilon}=[-\log \varepsilon-$ 
1], where $[a]$ denotes the integer part of $a$. Then by (S.6),

$$
\begin{aligned}
& P\left(\sup _{g \in \mathcal{G}} \frac{\sqrt{n}\left\|Z_{n}(g)\right\|}{a_{n}\|g\|_{\text {sup }}^{\gamma}+1} \geq T_{n}\right) \leq P\left(\sup _{\substack{g \in \mathcal{G} \\
\|g\|_{\text {sup }} \leq \varepsilon^{1 / \gamma}}} \frac{\sqrt{n}\left\|Z_{n}(g)\right\|}{a_{n}\|g\|_{\text {sup }}^{\gamma}+1} \geq T_{n}\right) \\
& +\sum_{l=0}^{Q_{\varepsilon}} P\left(\sup _{\substack{g \in \mathcal{G} \\
\left(2^{l} \varepsilon\right)^{1 / \gamma} \leq\|g\|_{\text {sup }} \leq\left(2^{l+1} \varepsilon\right)^{1 / \gamma}}} \frac{\sqrt{n}\left\|Z_{n}(g)\right\|}{a_{n}\|g\|_{\text {sup }}^{\gamma}+1} \geq T_{n}\right) \\
& \leq P\left(\sup _{\substack{g \in \mathcal{G} \\
\|g\|_{\mathrm{sup}} \leq \varepsilon^{1 / \gamma}}} \sqrt{n}\left\|Z_{n}(g)\right\| \geq T_{n}\right) \\
& +\sum_{l=0}^{Q_{\varepsilon}} P\left(\sup _{\substack{g \in \mathcal{G} \\
\|g\|_{\sup } \leq\left(2^{l+1} \varepsilon\right)^{1 / \gamma}}} \sqrt{n}\left\|Z_{n}(g)\right\| \geq\left(1+2^{l}\right) T_{n}\right) \\
& \leq 2 \exp \left(-h^{(2 m-1) /(2 m)}\left(\varepsilon^{1 / \gamma}\right)^{-2+1 / m} T_{n}^{2} / n\right) \\
& +\sum_{l=0}^{Q_{\varepsilon}} 2 \exp \left(-h^{(2 m-1) /(2 m)}\left[\left(2^{l+1} \varepsilon\right)^{1 / \gamma}\right]^{-2+1 / m} T_{n}^{2}\left(2^{l}+1\right)^{2} / n\right) \\
& =2 \exp \left(-T_{n}^{2}\right)+\sum_{l=0}^{Q_{\varepsilon}} 2 \exp \left(-2^{-2(l+1)} T_{n}^{2}\left(2^{l}+1\right)^{2}\right) \\
& \leq 2\left(Q_{\varepsilon}+2\right) \exp \left(-T^{2} / 4\right) \leq \mathrm{const} \cdot \log n(\log n)^{-5 / 4} \rightarrow 0,
\end{aligned}
$$

as $n \rightarrow \infty$. This proves the result.

S.6. Proof of Proposition 3.3. To prove Proposition 3.3, we first need the following Lemma. Denote $N\left(\delta, \mathcal{G},\|\cdot\|_{\text {sup }}\right)$ as the $\delta$-covering number of the function class $\mathcal{G}$ in terms of the uniform norm.

Lemma S.1. Suppose that $c_{m}^{-2} h \lambda^{-1}>1$. Then, for any $\delta>0$, we have

$$
\log N\left(\delta, \mathcal{G},\|\cdot\|_{\mathrm{sup}}\right) \leq C\left(h \lambda^{-1}\right)^{1 /(2 m)} \delta^{-1 / m},
$$

where $C>0$ is an universal constant.

Proof of Lemma S.1. Note that by $c_{m}^{-2} h \lambda^{-1}>1$,

$$
\mathcal{G}=\left(c_{m}^{-2} h \lambda^{-1}\right)^{1 / 2} \cdot\left\{g \in \mathcal{H} \mid\|g\|_{\text {sup }} \leq\left(c_{m}^{-2} h \lambda^{-1}\right)^{-1 / 2}, J(g, g) \leq 1\right\} \subset\left(c_{m}^{-2} h \lambda^{-1}\right)^{1 / 2} \mathcal{T},
$$

where $\mathcal{T}=\left\{g \in \mathcal{H} \mid\|g\|_{\text {sup }} \leq 1, J(g, g) \leq 1\right\}$. So, by [27], we have

$$
\begin{aligned}
\log N\left(\delta, \mathcal{G},\|\cdot\|_{\text {sup }}\right) & \leq \log N\left(\delta,\left(c_{m}^{-2} h \lambda^{-1}\right)^{1 / 2} \mathcal{T},\|\cdot\|_{\text {sup }}\right) \\
& =\log N\left(\left(c_{m}^{-2} h \lambda^{-1}\right)^{-1 / 2} \delta, \mathcal{T},\|\cdot\|_{\text {sup }}\right) \\
& \leq c\left(\left(c_{m}^{-2} h \lambda^{-1}\right)^{-1 / 2} \delta\right)^{-1 / m}=c c_{m}^{-1 / m}\left(h \lambda^{-1}\right)^{1 /(2 m)} \delta^{-1 / m} .
\end{aligned}
$$


Consider the function class $\mathcal{F}=\left\{g \in \mathcal{H} \mid\|g\|_{\text {sup }} \leq 1, J(g, g) \leq 1\right\}$. By Lemma S.1, for any $\delta>0$, we know $\log N\left(\delta, \mathcal{F},\|\cdot\|_{\text {sup }}\right) \leq c \delta^{-1 / m}$, where $c$ is some universal constant. Then a modification of Lemma 3.2 leads to

Lemma S.2. Suppose that $\psi_{n}$ satisfies the following Lipschitz continuity condition, i.e.,

$$
\left|\psi_{n}(T ; f)-\psi_{n}(T ; g)\right| \leq c_{m}^{-1} h^{1 / 2}\|f-g\|_{\text {sup }}, \text { for all } f, g \in \mathcal{F}
$$

where $c_{m}$ is specified in Lemma 3.1. Then we have

$$
\lim _{n \rightarrow \infty} P\left(\sup _{g \in \mathcal{F}} \frac{\left\|Z_{n}(g)\right\|}{\|g\|_{\text {sup }}^{1-1 /(2 m)}+n^{-1 / 2}} \leq(5 \log \log n)^{1 / 2}\right)=1,
$$

where the empirical process $Z_{n}(g)$ is defined in (3.1).

Denote $g=\widehat{g}_{n, \lambda}-g_{0}$. By consistency of $\widehat{g}_{n, \lambda}$ in $\|\cdot\|_{\mathcal{H}}$-norm and Sobolev embedding Theorem (see [1]), we know that $\widehat{g}_{n, \lambda}(z)$ falls in $\mathcal{I}$ for any $z \in \mathbb{I}$ and large enough $n$. By Taylor's expansion,

$$
\ell_{n, \lambda}\left(g_{0}+g\right)-\ell_{n, \lambda}\left(g_{0}\right)=S_{n, \lambda}\left(g_{0}\right) g+\frac{1}{2} D S_{n, \lambda}\left(g_{0}\right) g g+\frac{1}{6} D^{2} S_{n, \lambda}\left(g^{\star}\right) g g g \geq 0,
$$

where $g^{\star}=g_{0}+t^{\star} g$ for some $t^{\star} \in[0,1]$. Denote the three terms on the right side of the above equation by $I_{1}, I_{2}, I_{3}$. Next we will study the rates for these terms. Denote $A_{i}=\left\{\sup _{a \in \mathcal{I}}\left|\ddot{\ell}_{a}\left(Y_{i} ; a\right)\right|+\right.$ $\left.\sup _{a \in \mathcal{I}}\left|\ell_{a}^{\prime \prime \prime}\left(Y_{i} ; a\right)\right| \leq C \log n\right\}$. By (2.4), we may choose $C$ to be large so that $\cap_{i} A_{i}$ has large probability and $P\left(A_{i}^{c}\right)=O\left(n^{-2}\right)$. Then, on $\cap_{i} A_{i}$, we have

$$
\begin{aligned}
\left|6 I_{3}\right| & \leq \frac{1}{n} \sum_{i=1}^{n} \sup _{a \in \mathcal{I}}\left|\ell_{a}^{\prime \prime \prime}\left(Y_{i} ; a\right)\right| \cdot\left|g\left(Z_{i}\right)\right|^{3} \\
& \leq \frac{1}{n}\|g\|_{\sup } \sum_{i=1}^{n} \sup _{a \in \mathcal{I}}\left|\ell_{a}^{\prime \prime \prime}\left(Y_{i} ; a\right)\right| \cdot g\left(Z_{i}\right)^{2} \\
& =\frac{1}{n}\|g\|_{\sup }\left\langle\sum_{i=1}^{n} \psi\left(T_{i} ; g\right) K_{Z_{i}}, g\right\rangle \\
& =\frac{1}{n}\|g\|_{\sup }\left\langle\sum_{i=1}^{n}\left[\psi\left(T_{i} ; g\right) K_{Z_{i}}-E\left\{\psi(T ; g) K_{Z}\right\}\right], g\right\rangle+\|g\|_{\sup } E\{\psi(T ; g) g(Z)\}
\end{aligned}
$$

where $\psi\left(T_{i} ; g\right)=\sup _{a \in \mathcal{I}}\left|\ell_{a}^{\prime \prime \prime}\left(Y_{i} ; a\right)\right| g\left(Z_{i}\right) I_{A_{i}}$. Let $\psi_{n}\left(T_{i} ; g\right)=(C \log n)^{-1} c_{m}^{-1} h^{1 / 2} \psi\left(T_{i} ; g\right)$, which satisfies (S.7). Thus, by Lemma S.2, we have, with large probability give large $n$,

$$
\left\|\sum_{i=1}^{n}\left[\psi_{n}\left(T_{i} ; g\right) K_{Z_{i}}-E\left\{\psi_{n}(T ; g) K_{Z}\right\}\right]\right\| \leq\left(n^{1 / 2}\|g\|_{\sup }^{1-1 /(2 m)}+1\right)(5 \log \log n)^{1 / 2} .
$$


So, by Cauchy's inequality, we have

$$
\left|\left\langle\sum_{i=1}^{n}\left[\psi\left(T_{i} ; g\right) K_{Z_{i}}-E\left\{\psi(T ; g) K_{Z}\right\}\right], g\right\rangle\right| \leq\|g\| \cdot\left(n^{1 / 2}\|g\|_{\sup }^{1-1 /(2 m)}+1\right)(5 \log \log n)^{1 / 2} .
$$

On the other hand, by Assumption A.1 (a), we have

$$
E\{\psi(T ; g) g(Z)\} \leq E\left\{\sup _{a \in \mathcal{I}}\left|\ell_{a}^{\prime \prime \prime}(Y ; a)\right| g(Z)^{2}\right\} \leq 2 C_{0}^{2} C_{1}\|g\|^{2} .
$$

By $\left(n^{1 / 2} h\right)^{-1}(\log \log n)^{m /(2 m-1)}(\log n)^{2 m /(2 m-1)}=o(1)$, which implies $\left(n^{1 / 2} h\right)^{-1}(\log \log n)^{1 / 2} \log n=$ $o(1)$, we have

$$
\begin{aligned}
\left|6 I_{3}\right| & \leq \frac{1}{n}\|g\|_{\text {sup }} \cdot\|g\|(C \log n) c_{m} h^{-1 / 2}\left(n^{1 / 2}\|g\|_{\text {sup }}^{1-1 /(2 m)}+1\right)(5 \log \log n)^{1 / 2}+2 C_{0}^{2} C_{1}\|g\|_{\text {sup }} \cdot\|g\|^{2} \\
& =c_{m}^{2} C^{\prime}\left(n^{1 / 2} h\right)^{-1}(\log \log n)^{1 / 2}(\log n)\|g\|^{2}+2 C_{0}^{2} C_{1}\|g\|_{\text {sup }} \cdot\|g\|^{2} \\
(\mathrm{~S} .8) & =o_{P}(1) \cdot\|g\|^{2} .
\end{aligned}
$$

To approximate $I_{2}$, by Cauchy's inequality, we have

$$
\begin{aligned}
\left|E\left\{\ddot{\ell}_{a}\left(Y ; g_{0}(Z)\right) I_{A^{c}} g(Z)^{2}\right\}\right| & \leq E\left\{\left|\ddot{\ell}_{a}\left(Y ; g_{0}(Z)\right)\right|^{2} I_{A^{c}} g(Z)^{4}\right\}^{1 / 2} \cdot P\left(A^{c}\right)^{1 / 2} \\
& \leq O(1) \cdot(\log n)\|g\|_{\text {sup }}\|g\| n^{-1}=\|g\|^{2} O\left((n h)^{-1 / 2}\right)=o(1)\|g\|^{2} .
\end{aligned}
$$

By changing $\psi$ and $\psi_{n}$ in the proof of (S.8) to $\psi\left(T_{i} ; g\right)=\ddot{\ell}_{a}\left(Y_{i} ; g_{0}\left(Z_{i}\right)\right) g I_{A_{i}}$ and $\psi_{n}\left(T_{i} ; g\right)=$ $(C \log n)^{-1} c_{m}^{-1} h^{1 / 2} \psi\left(T_{i} ; g\right)$, and using an argument similar to the proof of (S.8), we have

$$
\begin{aligned}
\left|\left[D S_{n, \lambda}\left(g_{0}\right)-E\left\{D S_{n, \lambda}\left(g_{0}\right)\right\}\right] g g\right| \leq & C c_{m} h^{-1+1 /(4 m)} n^{-1 / 2}(\log \log n)^{1 / 2}(\log n)\|g\|^{2-1 /(2 m)} \\
& +C c_{m}\left(n h^{1 / 2}\right)^{-1}(\log \log n)^{1 / 2}(\log n)\|g\|+o_{P}(1)\|g\|^{2} .
\end{aligned}
$$

Thus,

$$
\begin{aligned}
2 I_{2}= & -\|g\|^{2}+C c_{m} h^{-1+1 /(4 m)} n^{-1 / 2}(\log \log n)^{1 / 2}(\log n)\|g\|^{2-1 /(2 m)} \\
& +C c_{m}\left(n h^{1 / 2}\right)^{-1}(\log \log n)^{1 / 2}(\log n)\|g\|+o_{P}(1)\|g\|^{2} .
\end{aligned}
$$

Note that $E\left\{\left\|\sum_{i=1}^{n} \epsilon_{i} K_{Z_{i}}\right\|^{2}\right\}=O\left(n h^{-1}\right)$. By (S.44) in the main paper, we have

$$
\left\|S_{n, \lambda}\left(g_{0}\right)\right\|=O_{P}\left((n h)^{-1 / 2}+\lambda^{1 / 2}\right) .
$$

Combining (S.8), (S.9), and (S.10), and by $\left(n h^{1 / 2}\right)^{-1}(\log \log n)^{1 / 2}(\log n)=o\left((n h)^{-1 / 2}\right)$, we have for some large $C^{\prime}$

$$
\left(1+o_{P}(1)\right)\|g\|^{2} \leq C^{\prime}\left((n h)^{-1 / 2}+\lambda^{1 / 2}\right)\|g\|+C c_{m} h^{-1+1 /(4 m)} n^{-1 / 2}(\log \log n)^{1 / 2}(\log n)\|g\|^{2-1 /(2 m)} .
$$

Solving this inequality, and using $\left(n^{1 / 2} h\right)^{-1}(\log \log n)^{m /(2 m-1)}(\log n)^{2 m /(2 m-1)}=o(1)$, we get $\|g\|=O_{P}\left((n h)^{-1 / 2}+\lambda^{1 / 2}\right)$. 
S.7. Proof of Theorem 3.4. By Assumption A.1 (a), it is not difficult to check the following

$$
\max _{1 \leq i \leq n} \sup _{a \in \mathcal{I}}\left|\ddot{\ell}_{a}\left(Y_{i} ; a\right)\right|=O_{P}(\log n)
$$

By (S.11), we can let $C>C_{0}$ be sufficiently large so that the event $B_{n 1}=\left\{\max _{1 \leq i \leq n} \sup _{a \in \mathcal{I}}\left|\ddot{\ell}_{a}\left(Y_{i} ; a\right)\right| \leq\right.$ $C \log n\}$ has large probability. Denote $g=\widehat{g}_{n, \lambda}-g_{0}$. By Assumption A.3, the event $B_{n 2}=$ $\left\{\|g\| \leq r_{n} \equiv M\left((n h)^{-1 / 2}+h^{m}\right)\right\}$ has large probability with some pre-selected large $M$. So $B_{n}=B_{n 1} \cap B_{n 2}$ has a large probability. Define $\widetilde{g}=d_{n}^{-1} g$, where $d_{n}=c_{m} r_{n} h^{-1 / 2}$. Since $h=o(1)$ and $n h^{2} \rightarrow \infty, d_{n}=o(1)$. Then by Lemma 3.1, on $B_{n}$, $\|\widetilde{g}\|_{\text {sup }} \leq 1$. Note that $J(\widetilde{g}, \widetilde{g})=d_{n}^{-2} \lambda^{-1}(\lambda J(g, g)) \leq d_{n}^{-2} \lambda^{-1}\|g\|^{2} \leq d_{n}^{-2} \lambda^{-1} r_{n}^{2} \leq c_{m}^{-2} h \lambda^{-1}$. Thus, when the event $B_{n}$ holds, $\widetilde{g}$ is an element in $\mathcal{G}$.

Define $\psi(T ; g)=\dot{\ell}_{a}\left(Y ; g(Z)+g_{0}(Z)\right)-\dot{\ell}_{a}\left(Y ; g_{0}(Z)\right)$. By the definition of $S_{n}$ and a direct calculation, one can verify that $S_{n}\left(g+g_{0}\right)-S\left(g+g_{0}\right)-\left(S_{n}\left(g_{0}\right)-S\left(g_{0}\right)\right)=\frac{1}{n} \sum_{i=1}^{n}\left[\psi\left(T_{i} ; g\right) K_{Z_{i}}-\right.$ $\left.E\left\{\psi(T ; g) K_{Z}\right\}\right]$. Let $\widetilde{\psi}_{n}(T ; \widetilde{g})=C^{-1} c_{m}^{-1}(\log n)^{-1} h^{1 / 2} d_{n}^{-1} \psi\left(T ; d_{n} \widetilde{g}\right)$ and $\psi_{n}\left(T_{i} ; \widetilde{g}\right)=\widetilde{\psi}_{n}\left(T_{i} ; \widetilde{g}\right) I_{A_{i}}$, where $A_{i}=\left\{\sup _{a \in \mathcal{I}}\left|\ddot{\ell}_{a}\left(Y_{i} ; a\right)\right| \leq C \log n\right\}$ for $i=1, \ldots, n$. Observe that $B_{n}$ implies $\cap_{i} A_{i}$.

Next we show that $\psi_{n}$ satisfies (3.2). For any $g_{1}, g_{2} \in \mathcal{G}$, and $z \in \mathbb{I}$, both $g_{0}(z)+d_{n} g_{1}(z)$ and $g_{0}(z)+d_{n} g_{2}(z)$ fall in $\mathcal{I}$ when $n$ is sufficiently large since $g_{0}(z) \in \mathcal{I}_{0}$ and $d_{n}=o(1)$. Recall that $\mathcal{I}_{0}$ and $\mathcal{I}$ are specified in Assumption A.1. Therefore,

$$
\begin{aligned}
\left|\psi_{n}\left(T_{i} ; d_{n} g_{1}\right)-\psi_{n}\left(T_{i} ; d_{n} g_{2}\right)\right|= & C^{-1} c_{m}^{-1}(\log n)^{-1} h^{1 / 2} d_{n}^{-1}\left|\psi\left(T_{i} ; g_{1}\right)-\psi\left(T_{i} ; g_{2}\right)\right| \cdot I_{A_{i}} \\
= & C^{-1} c_{m}^{-1}(\log n)^{-1} h^{1 / 2} d_{n}^{-1} \mid \int_{g_{0}\left(Z_{i}\right)}^{g_{0}\left(Z_{i}\right)+d_{n} g_{1}\left(Z_{i}\right)} \ddot{\ell}_{a}\left(Y_{i} ; a\right) \cdot I_{A_{i}} d a \\
& -\int_{g_{0}\left(Z_{i}\right)}^{g_{0}\left(Z_{i}\right)+d_{n} g_{1}\left(Z_{i}\right)} \ddot{\ell}_{a}\left(Y_{i} ; a\right) \cdot I_{A_{i}} d a \mid \\
\leq & C^{-1} c_{m}^{-1}(\log n)^{-1} h^{1 / 2} d_{n}^{-1} \cdot d_{n}\left\|g_{1}-g_{2}\right\|_{\text {sup }} \cdot \sup _{a \in \mathcal{I}}\left|\ddot{\ell}_{a}\left(Y_{i} ; a\right)\right| \cdot I_{A_{i}} \\
\leq & C^{-1} c_{m}^{-1}(\log n)^{-1} h^{1 / 2} d_{n}^{-1} \cdot d_{n} \cdot C \log n \cdot\left\|g_{1}-g_{2}\right\|_{\text {sup }} \\
= & c_{m}^{-1} h^{1 / 2}\left\|g_{1}-g_{2}\right\|_{\text {sup }} .
\end{aligned}
$$

Thus, $\psi_{n}$ satisfies (3.2). By Lemma 3.2, with large probability

$$
\left\|\sum_{i=1}^{n}\left[\psi_{n}\left(T_{i} ; \widetilde{g}\right) K_{Z_{i}}-E\left\{\psi_{n}(T ; \widetilde{g}) K_{Z}\right\}\right]\right\| \leq\left(n^{1 / 2} h^{-(2 m-1) /(4 m)}+1\right)(5 \log \log n)^{1 / 2} .
$$

On the other hand, by Chebyshev's inequality

$$
P\left(A_{i}^{c}\right)=\exp \left(-\left(C / C_{0}\right) \log n\right) E\left\{\exp \left(\sup _{a \in \mathcal{I}}\left|\ddot{\ell}_{a}\left(Y_{i} ; a\right)\right| / C_{0}\right)\right\} \leq C_{1} n^{-C / C_{0}}
$$

Since $h=o(1)$ and $n h^{2} \rightarrow \infty$, we may choose $C$ to be large enough so that $2^{1 / 2} C^{-1} C_{0} C_{1}(\log n)^{-1} n^{-C /\left(2 C_{0}\right)}<$ $a_{n}^{\prime} h^{1 / 2} d_{n}^{-1}$, where $a_{n}^{\prime}=n^{-1 / 2}\left((n h)^{-1 / 2}+h^{m}\right) h^{-(6 m-1) /(4 m)}(\log \log n)^{1 / 2}$. By (2.3), which implies 
$E\left\{\sup _{a \in \mathcal{I}}\left|\ddot{\ell}_{a}\left(Y_{i} ; a\right)\right| \mid Z_{i}\right\} \leq 2 C_{1} C_{0}^{2}$, we have $E\left\{\left|\psi\left(T ; d_{n} \widetilde{g}\right)\right|^{2}\right\} \leq 2 C_{1} C_{0}^{2} d_{n}^{2}$ on $B_{n}$, where the expectation is taken with respect to $T=(Y, Z)$. So when $n$ is large, by Chebyshev's inequality, we have on $B_{n}$,

$$
\begin{aligned}
\left\|E\left\{\psi_{n}\left(T_{i} ; \widetilde{g}\right) K_{Z_{i}}\right\}-E\left\{\widetilde{\psi}_{n}\left(T_{i} ; \widetilde{g}\right) K_{Z_{i}}\right\}\right\| & =\left\|E\left\{\widetilde{\psi}_{n}\left(T_{i} ; \widetilde{g}\right) K_{Z_{i}} \cdot I_{A_{i}^{c}}\right\}\right\| \\
& \leq C^{-1}(\log n)^{-1} d_{n}^{-1}\left(E\left\{\left|\psi\left(T_{i} ; d_{n} \widetilde{g}\right)\right|^{2}\right\}\right)^{1 / 2} P\left(A_{i}^{c}\right)^{1 / 2} \\
& \leq 2^{1 / 2} C^{-1} C_{0} C_{1}(\log n)^{-1} n^{-C /\left(2 C_{0}\right)} \\
& \leq a_{n}^{\prime} h^{1 / 2} d_{n}^{-1},
\end{aligned}
$$

where the expectation is taken with respect to $T_{i}$. Therefore, by (S.12) and on $B_{n}$, we have

$$
\begin{aligned}
& \left\|S_{n}\left(g+g_{0}\right)-S\left(g+g_{0}\right)-\left(S_{n}\left(g_{0}\right)-S\left(g_{0}\right)\right)\right\| \\
= & \frac{C c_{m}(\log n) h^{-1 / 2} d_{n}}{n}\left\|\sum_{i=1}^{n}\left[\widetilde{\psi}_{n}\left(T_{i} ; \widetilde{g}\right) K_{Z_{i}}-E\left\{\widetilde{\psi}_{n}(T ; \widetilde{g}) K_{Z}\right\}\right]\right\| \\
\leq & \frac{C c_{m}(\log n) h^{-1 / 2} d_{n}}{n}\left(\left\|\sum_{i=1}^{n}\left[\psi_{n}\left(T_{i} ; \widetilde{g}\right) K_{Z_{i}}-E\left\{\psi_{n}(T ; \widetilde{g}) K_{Z}\right\}\right]\right\|\right. \\
& \left.+n\left\|E\left\{\psi_{n}\left(T_{i} ; \widetilde{g}\right) K_{Z_{i}}\right\}-E\left\{\widetilde{\psi}_{n}\left(T_{i} ; \widetilde{g}\right) K_{Z_{i}}\right\}\right\|\right) \\
\leq & \frac{C c_{m}(\log n) h^{-1 / 2} d_{n}}{n} \cdot\left[\left(n^{1 / 2} h^{-(2 m-1) /(4 m)}+1\right)(5 \log \log n)^{1 / 2}+n a_{n}^{\prime} h^{1 / 2} d_{n}^{-1}\right] \\
\leq & C^{\prime} c_{m} a_{n}^{\prime} \log n,
\end{aligned}
$$

for some constant $C^{\prime}>0$ that only depends on $C, c_{m}, M$.

By Taylor's expansion, the fact $S_{n, \lambda}\left(g+g_{0}\right)=0$, and Proposition 2.3, we have

$$
\begin{aligned}
& \left\|S_{n}\left(g+g_{0}\right)-S\left(g+g_{0}\right)-\left(S_{n}\left(g_{0}\right)-S\left(g_{0}\right)\right)\right\| \\
= & \left\|S_{n, \lambda}\left(g+g_{0}\right)-S_{\lambda}\left(g+g_{0}\right)-S_{n, \lambda}\left(g_{0}\right)+S_{\lambda}\left(g_{0}\right)\right\| \\
= & \left\|S_{\lambda}\left(g+g_{0}\right)+S_{n, \lambda}\left(g_{0}\right)-S_{\lambda}\left(g_{0}\right)\right\| \\
= & \left\|D S_{\lambda}\left(g_{0}\right) g+\int_{0}^{1} \int_{0}^{1} s D^{2} S_{\lambda}\left(g_{0}+s s^{\prime} g\right) g g d s d s^{\prime}+S_{n, \lambda}\left(g_{0}\right)\right\| \\
= & \left\|-g+\int_{0}^{1} \int_{0}^{1} s D^{2} S_{\lambda}\left(g_{0}+s s^{\prime} g\right) g g d s d s^{\prime}+S_{n, \lambda}\left(g_{0}\right)\right\| \\
\geq & \left\|-g+S_{n, \lambda}\left(g_{0}\right)\right\|-\left\|\int_{0}^{1} \int_{0}^{1} s D^{2} S_{\lambda}\left(g_{0}+s s^{\prime} g\right) g g d s d s^{\prime}\right\| .
\end{aligned}
$$

Therefore,

$$
\begin{aligned}
& \left\|g-S_{n, \lambda}\left(g_{0}\right)\right\| \\
\leq & \left\|S_{n}\left(g+g_{0}\right)-S\left(g+g_{0}\right)-\left(S_{n}\left(g_{0}\right)-S\left(g_{0}\right)\right)\right\|+\left\|\int_{0}^{1} \int_{0}^{1} s D^{2} S_{\lambda}\left(g_{0}+s s^{\prime} g\right) g g d s d s^{\prime}\right\| \\
(\mathrm{S} .14) \leq & \left\|S_{n}\left(g+g_{0}\right)-S\left(g+g_{0}\right)-\left(S_{n}\left(g_{0}\right)-S\left(g_{0}\right)\right)\right\|+\int_{0}^{1} \int_{0}^{1} s\left\|D^{2} S_{\lambda}\left(g_{0}+s s^{\prime} g\right) g g\right\| d s d s^{\prime} .
\end{aligned}
$$


Next we find an upper bound for $\left\|D^{2} S_{\lambda}\left(g_{0}+s s^{\prime} g\right) g g\right\|$. The Fréchet derivative of $D S_{\lambda}$ is found to be $D^{2} S_{\lambda}=D^{2} S$ Therefore, $D^{2} S_{\lambda}\left(g_{0}+s s^{\prime} g\right) g g=D^{2} S\left(g_{0}+s s^{\prime} g\right) g g=E\left\{\ell_{a}^{\prime \prime \prime}\left(Y ;\left(g_{0}+\right.\right.\right.$ $\left.\left.s s^{\prime} g\right)(Z)\right) g(Z)^{2} K_{Z}$, where the expectation is taken with respect to $T$. Hence, by (2.4) and on $B_{n}$, we have

$$
\begin{aligned}
\left\|D^{2} S_{\lambda}\left(g_{0}+s s^{\prime} g\right) g g\right\|=\left\|E\left\{\ell_{a}^{\prime \prime \prime}\left(Y ;\left(g_{0}+s s^{\prime} g\right)(Z)\right) g(Z)^{2} K_{Z}\right\}\right\| & \leq E\left\{E\left\{\sup _{a \in \mathcal{I}} \mid \ell_{a}^{\prime \prime \prime}(Y ; a) \| Z\right\} g(Z)^{2}\left\|K_{Z}\right\|\right\} \\
(\mathrm{S} .15) & \leq C_{\ell} c_{m} h^{-1 / 2}\|g\|^{2},
\end{aligned}
$$

where $C_{\ell}=\sup _{z \in \mathbb{I}} E\left\{\sup _{a \in \mathcal{I}} \mid \ell_{a}^{\prime \prime \prime}(Y ; a) \| Z=z\right\}$. Thus, from (S.13), (S.14) and (S.15), with large probability, $\left\|g-S_{n, \lambda}\left(g_{0}\right)\right\| \leq C^{\prime} c_{m} a_{n}^{\prime} \log n+C_{\ell} c_{m} h^{-1 / 2}\left((n h)^{-1 / 2}+h^{m}\right)^{2}$. This completes the proof of Theorem 3.4.

S.8. Proof of Theorem 3.5. Define $\operatorname{Rem}_{n}=\widehat{g}_{n, \lambda}-g_{0}^{*}-\frac{1}{n} \sum_{i=1}^{n} \epsilon_{i} K_{Z_{i}}$. By Theorem 3.4, $\operatorname{Rem}_{n}$ satisfies $\left\|\operatorname{Rem}_{n}\right\|=O_{P}\left(a_{n} \log n\right)$. By assumption $a_{n} \log n=o\left(n^{-1 / 2}\right)$, we have $\left\|\operatorname{Re} m_{n}\right\|=$ $o_{P}\left(n^{-1 / 2}\right)$. Since $E\left\{\left\|\sum_{i=1}^{n} \epsilon_{i} K_{Z_{i}}\right\|^{2}\right\}=n E\left\{\epsilon^{2}\left\|K_{Z}\right\|^{2}\right\}=O\left(n h^{-1}\right)$, we have $\left\|n^{-1} \sum_{i=1}^{n} \epsilon_{i} K_{Z_{i}}\right\|=$ $O_{P}\left((n h)^{-1 / 2}\right)$. Thus, $\operatorname{Rem}_{n}$ is negligible compared with $\sum_{i=1}^{n} \epsilon_{i} K_{Z_{i}}$.

Next we show the limiting distribution of $(n h)^{1 / 2}\left(\widehat{g}_{n, \lambda}\left(z_{0}\right)-g_{0}^{*}\left(z_{0}\right)\right)$. Note that this is equal to $(n h)^{1 / 2}\left\langle K_{z_{0}}, \widehat{g}_{n, \lambda}-g_{0}^{*}\right\rangle$. Using the fact

$$
\begin{aligned}
\left|(n h)^{1 / 2}\left\langle K_{z_{0}}, \widehat{g}_{n, \lambda}-g_{0}^{*}-\frac{1}{n} \sum_{i=1}^{n} \epsilon_{i} K_{Z_{i}}\right\rangle\right| & \leq(n h)^{1 / 2}\left\|K_{z_{0}}\right\| \cdot\left\|\operatorname{Rem}_{n}\right\| \\
& =O_{P}\left((n h)^{1 / 2} h^{-1 / 2} a_{n} \log n\right)=o_{P}(1)
\end{aligned}
$$

we just need to find the limiting distribution of $(n h)^{1 / 2}\left\langle K_{z_{0}}, \frac{1}{n} \sum_{i=1}^{n} \epsilon_{i} K_{Z_{i}}\right\rangle=\left(n h^{-1}\right)^{-1 / 2} \sum_{i=1}^{n} \epsilon_{i} K_{Z_{i}}\left(z_{0}\right)$.

By Assumption A.1 (c), i.e., $E\left\{\epsilon^{2} \mid Z\right\}=I(Z)$, we have

$\operatorname{Var}\left(\sum_{i=1}^{n} \epsilon_{i} K_{Z_{i}}\left(z_{0}\right)\right)=n E\left\{\epsilon^{2}\left|K_{Z}\left(z_{0}\right)\right|^{2}\right\}=n E\left\{E\left\{\epsilon^{2} \mid Z\right\}\left|K_{Z}\left(z_{0}\right)\right|^{2}\right\}=n E\left\{I(Z)\left|K_{Z}\left(z_{0}\right)\right|^{2}\right\}=n V\left(K_{z_{0}}, K_{z_{0}}\right)$.

By the definition, as $h \rightarrow 0, h V\left(K_{z_{0}}, K_{z_{0}}\right) \rightarrow \sigma_{z_{0}}^{2}$. Thus, $\left(n h^{-1}\right)^{-1 / 2} \sum_{i=1}^{n} \epsilon_{i} K_{Z_{i}}\left(z_{0}\right) \stackrel{d}{\longrightarrow} N\left(0, \sigma_{z_{0}}^{2}\right)$ by CLT. The expression of $\sigma_{z_{0}}^{2}$, i.e., (3.8), follows from Proposition 2.1. This completes the proof.

S.9. Proof of Corollary 3.7. By Proposition 2.2, Assumption A.2 holds. We first show part (i). By $\ell_{a}^{\prime \prime \prime}(y ; a)=0$ for any $y$ and $a$, i.e., , in (3.5) $C_{\ell}=0$, we obtain $a_{n}=n^{-1 / 2}\left((n h)^{-1 / 2}+\right.$ $\left.h^{m}\right) h^{-(6 m-1) /(4 m)}(\log \log n)^{1 / 2}$. Since $h \asymp n^{-1 /(4 m+1)}$, we have $h=o(1)$ and $n h^{2} \rightarrow \infty$. By $m>(3+\sqrt{5}) / 4$, it can be verified that $a_{n} \log n=o\left(n^{-1 / 2}\right)$.

On the other hand, by expression of $K$ in terms of $h_{\nu}$ (see Proposition 2.1), as $h \rightarrow 0$, we have

$$
\begin{aligned}
\int_{0}^{1} g_{0}^{(2 m)}(z) K\left(z_{0}, z\right) d z-g_{0}^{(2 m)}\left(z_{0}\right) / \pi\left(z_{0}\right) & =\sum_{\nu} \frac{1}{1+\lambda \gamma_{\nu}} V\left(g_{0}^{(2 m)} / \pi, h_{\nu}\right) h_{\nu}\left(z_{0}\right)-\sum_{\nu} V\left(g_{0}^{(2 m)} / \pi, h_{\nu}\right) h_{\nu}\left(z_{0}\right) \\
& =-\sum_{\nu} \frac{\lambda \gamma_{\nu}}{1+\lambda \gamma_{\nu}} V\left(g_{0}^{(2 m)} / \pi, h_{\nu}\right) h_{\nu}\left(z_{0}\right) \rightarrow 0
\end{aligned}
$$


where the limit in (S.16) follows from $\sum_{\nu}\left|V\left(g_{0}^{(2 m)}, h_{\nu}\right) h_{\nu}\left(z_{0}\right)\right|<\infty$ and the dominated convergence theorem. Then, by (3.11) and integration by parts, it can be shown that

$$
\begin{aligned}
& \left(W_{\lambda} g_{0}\right)\left(z_{0}\right)=\left\langle W_{\lambda} g_{0}, K_{z_{0}}\right\rangle=\lambda J\left(g_{0}, K_{z_{0}}\right) \\
= & (-1)^{m} h^{2 m} \int_{0}^{1} g_{0}^{(2 m)}(z) K\left(z_{0}, z\right) d z=(-1)^{m} h^{2 m}\left(g_{0}^{(2 m)}\left(z_{0}\right) / \pi\left(z_{0}\right)+o(1)\right) .
\end{aligned}
$$

So, as $n \rightarrow \infty,(n h)^{1 / 2}\left(W_{\lambda} g_{0}\right)\left(z_{0}\right) \rightarrow(-1)^{m} g_{0}^{(2 m)}\left(z_{0}\right) / \pi\left(z_{0}\right)$. Therefore all assumptions in Theorem 3.6 hold. Then (3.12) directly follows from (3.10).

The proof of (3.13) is similar to that of (3.12). One only notes, by (S.17) and $h \asymp n^{-d}$ for $\frac{1}{4 m+1}<d \leq \frac{2 m}{8 m-1},(n h)^{1 / 2}\left(W_{\lambda} g_{0}\right)\left(z_{0}\right)=O\left((n h)^{1 / 2} h^{2 m}\right)=o(1)$. Then (3.13) follows from (3.10).

The proof of part (ii) is similar in spirit to that of part (i). The only difference is that (S.17) should be replaced by the following, by integration by parts,

$$
\left(W_{\lambda} g_{0}\right)\left(z_{0}\right)=h^{2 m} \sum_{j=1}^{m}(-1)^{j-1}\left[\left.\left(\frac{\partial^{m-j}}{\partial z^{m-j}} K_{z_{0}}^{m-j}(z)\right) \cdot g_{0}^{(m+j-1)}(z)\right|_{0} ^{1}\right]+(-1)^{m} h^{2 m} \int_{0}^{1} g_{0}^{(2 m)}(z) K\left(z_{0}, z\right) d z
$$

since $g_{0}$ does not satisfy the boundary conditions. The first sum is $o\left(h^{2 m}\right)$ by (3.14). The second sum is $(-1)^{m} h^{2 m}\left(g_{0}^{(2 m)}\left(z_{0}\right) / \pi\left(z_{0}\right)+o(1)\right)$ by (S.16). Thus, $\left(W_{\lambda} g_{0}\right)\left(z_{0}\right)=(-1)^{m} h^{2 m} g_{0}^{(2 m)}\left(z_{0}\right) / \pi\left(z_{0}\right)+$ $o\left(h^{2 m}\right)$. Note this is not true for $z_{0}=0$ or 1 . Then the proof can be finished by similar arguments in the proof of part (i).

S.10. Proof of Theorem 4.3. The proof is similar to those in Theorem 3.4, so we only sketch the basic idea. Let $g=\widehat{g}_{n, \lambda}^{0}-g_{0}^{0}$. Assumption A.4 guarantees that with large probability, $\|g\| \leq$ $r_{n} \equiv M\left((n h)^{-1 / 2}+h^{m}\right)$ for a suitably large $M$. By modifying the proof of Lemma 3.2, we have the following lemma.

Lemma S.3. Suppose that $\psi_{n}$ satisfies the following Lipschitz continuity condition:

$$
\left|\psi_{n}\left(T ; g_{1}\right)-\psi_{n}\left(T ; g_{2}\right)\right| \leq c_{m}^{-1} h^{1 / 2}\left\|g_{1}-g_{2}\right\|_{\text {sup }}, \text { for all } g_{1}, g_{2} \in \mathcal{H}_{0}
$$

where $T=(Y, Z)$ denotes the full data variable. Then we have

$$
\lim _{n \rightarrow \infty} P\left(\sup _{g \in \mathcal{G}_{0}} \frac{\left\|Z_{n}^{0}(g)\right\|}{n^{1 / 2} h^{-(2 m-1) /(4 m)}\|g\|_{\text {sup }}^{1-1 /(2 m)}+1} \leq(5 \log \log n)^{1 / 2}\right)=1,
$$

where $\mathcal{G}_{0}=\left\{g \in \mathcal{H}_{0} \mid\|g\|_{\text {sup }} \leq 1, J(g, g) \leq c_{m}^{-2} h \lambda^{-1}\right\}$ and $Z_{n}^{0}(g)=\sum_{i=1}^{n}\left[\psi_{n}\left(T_{i} ; g\right) K_{Z_{i}}^{*}-E\left\{\psi_{n}(T ; g) K_{Z}^{*}\right\}\right]$.

By reexamining the proof of Theorem 3.4, we have $g \in \mathcal{G}_{0}$ and $\psi_{n}$ satisfies Lipschitz continuity (S.19) with large probability, where $\psi_{n}(T ; g)=C^{-1} c_{m}^{-1}(\log n)^{-1} h^{1 / 2} d_{n}^{-1}\left\{\dot{\ell}_{a}\left(Y ; g_{0}(Z)+d_{n} g(Z)\right)-\right.$ $\left.\dot{\ell}_{a}\left(Y ; g_{0}(Z)\right)\right\}$ and $d_{n}=c_{m} r_{n} h^{-1 / 2}$. This leads to, with large probability,

$$
\left\|\sum_{i=1}^{n}\left[\psi_{n}\left(T_{i} ; g\right) K_{Z_{i}}^{*}-E\left\{\psi_{n}(T ; g) K_{Z}^{*}\right\}\right]\right\| \leq\left(n^{1 / 2} h^{-(2 m-1) /(4 m)}+1\right)(5 \log \log n)^{1 / 2} .
$$


The remainder of the proof follows by (S.11), and by an argument similar to (S.13) - (S.15).

S.11. Proof of Theorem 4.4. For notational convenience, denote $\widehat{g}=\widehat{g}_{n, \lambda}, \widehat{g}^{0}=\widehat{g}_{n, \lambda}^{0}, g=$ $w_{0}+\widehat{g}^{0}-\widehat{g}$. By Assumptions A.3 and A.4, with large probability, $\|g\|=O_{P}\left(r_{n}\right)$, where $r_{n}=$ $M\left((n h)^{-1 / 2}+h^{m}\right)$ for some large $M$. By Assumption A.1 (a), for some large constant $C>0$, the event $B_{n 1} \cap B_{n 2}$ has large probability, where $B_{n 1}=\left\{\max _{1 \leq i \leq n} \sup _{a \in \mathcal{I}}\left|\ddot{\ell}_{a}\left(Y_{i} ; a\right)\right| \leq C \log n\right\}$ and $B_{n 2}=\left\{\max _{1 \leq i \leq n} \sup _{a \in \mathcal{I}}\left|\ell_{a}^{\prime \prime \prime}\left(Y_{i} ; a\right)\right| \leq C \log n\right\}$. Let $a_{n}$ be defined as in (3.5).

By Taylor expansion,

$$
\begin{aligned}
L R T_{n, \lambda}= & \ell_{n, \lambda}\left(w_{0}+\widehat{g}^{0}\right)-\ell_{n, \lambda}(\widehat{g}) \\
= & S_{n, \lambda}(\widehat{g}) g+\int_{0}^{1} \int_{0}^{1} s D S_{n, \lambda}\left(\widehat{g}+s s^{\prime} g\right) g g d s d s^{\prime} \\
= & \int_{0}^{1} \int_{0}^{1} s D S_{n, \lambda}\left(\widehat{g}+s s^{\prime} g\right) g g d s d s^{\prime} \\
= & \int_{0}^{1} \int_{0}^{1} s\left\{D S_{n, \lambda}\left(\widehat{g}+s s^{\prime} g\right) g g-D S_{n, \lambda}\left(g_{0}\right) g g\right\} d s d s^{\prime} \\
& +\frac{1}{2}\left(D S_{n, \lambda}\left(g_{0}\right) g g-E\left\{D S_{n, \lambda}\left(g_{0}\right) g g\right\}\right)+\frac{1}{2} E\left\{D S_{n, \lambda}\left(g_{0}\right) g g\right\} .
\end{aligned}
$$

Denote the above three terms by $I_{1}, I_{2}$ and $I_{3}$. Next we will study the asymptotic behavior of these terms. Denote $\widetilde{g}=\widehat{g}+s s^{\prime} g-g_{0}$, for any $0 \leq s, s^{\prime} \leq 1$. So $\|\widetilde{g}\|=O_{P}\left(r_{n}\right)$.

We first study $I_{1}$. By calculations of the Fréchet derivatives, we have

$$
D S_{n, \lambda}\left(\widehat{g}+s s^{\prime} g\right) g g=D S_{n, \lambda}\left(\widetilde{g}+g_{0}\right) g g=\frac{1}{n} \sum_{i=1}^{n} \ddot{\ell}_{a}\left(Y_{i} ; g_{0}\left(Z_{i}\right)+\widetilde{g}\left(Z_{i}\right)\right) g\left(Z_{i}\right)^{2}-\left\langle W_{\lambda} g, g\right\rangle / 2,
$$

and $D S_{n, \lambda}\left(g_{0}\right) g g=\frac{1}{n} \sum_{i=1}^{n} \ddot{\ell}_{a}\left(Y_{i} ; g_{0}\left(Z_{i}\right)\right) g\left(Z_{i}\right)^{2}-\left\langle W_{\lambda} g, g\right\rangle / 2$. On $B_{n 1} \cap B_{n 2}$,

$$
\begin{aligned}
& \left|D S_{n, \lambda}\left(\widehat{g}+s s^{\prime} g\right) g g-D S_{n, \lambda}\left(g_{0}\right) g g\right| \\
\leq & \frac{1}{n} C(\log n)\|\widetilde{g}\|_{\sup } \sum_{i=1}^{n} g\left(Z_{i}\right)^{2} \\
= & C(\log n)\|\widetilde{g}\|_{\sup }\left\langle\frac{1}{n} \sum_{i=1}^{n} g\left(Z_{i}\right) K_{Z_{i}}, g\right\rangle \\
(\mathrm{S} .22)= & C(\log n)\|\widetilde{g}\|_{\sup }\left\langle\frac{1}{n} \sum_{i=1}^{n} g\left(Z_{i}\right) K_{Z_{i}}-E\left\{g(Z) K_{Z}\right\}, g\right\rangle+C(\log n)\|\widetilde{g}\|_{\sup } E\left\{g(Z)^{2}\right\},
\end{aligned}
$$

where the expectations are taken with respect to $Z$. Now we examine $\frac{1}{n} \| \sum_{i=1}^{n} g\left(Z_{i}\right) K_{Z_{i}}-$ $E\left\{g(Z) K_{Z}\right\} \|$. Let $d_{n}=c_{m} h^{-1 / 2} r_{n}$ and $\bar{g}=d_{n}^{-1} g$. Consider $\psi(T ; g)=g(Z)$ and $\psi_{n}(T ; \bar{g})=$ $c_{m}^{-1} h^{1 / 2} d_{n}^{-1} \psi\left(T ; d_{n} \bar{g}\right)$ (which satisfies (3.2)). Then by Lemma 3.2,

$\left\|\frac{1}{n} \sum_{i=1}^{n}\left[g\left(Z_{i}\right) K_{Z_{i}}-E\left\{g(Z) K_{Z}\right\}\right]\right\|=\frac{c_{m} h^{-1 / 2} d_{n}}{n}\left\|\sum_{i=1}^{n}\left[\psi_{n}\left(T_{i} ; \bar{g}\right) K_{Z_{i}}-E\left\{\psi_{n}(T ; \bar{g}) K_{Z}\right\}\right]\right\|=O_{P}\left(a_{n}^{\prime}\right)$, 
where $a_{n}^{\prime}=n^{-1 / 2}\left((n h)^{-1 / 2}+h^{m}\right) h^{-(6 m-1) /(4 m)}(\log \log n)^{1 / 2}$. Obviously, $E\left\{g(Z)^{2}\right\}=O\left(\|g\|^{2}\right)=$ $O_{P}\left(r_{n}^{2}\right)$. So, by $a_{n}^{\prime}=o\left(r_{n}\right)$, we have

$$
\begin{aligned}
\left|D S_{n, \lambda}\left(\widehat{g}+s s^{\prime} g\right) g g-D S_{n, \lambda}\left(g_{0}\right) g g\right| & =\|\widetilde{g}\|_{\sup }\left(O_{P}\left(a_{n}^{\prime} r_{n} \log n\right)+O_{P}\left(r_{n}^{2} \log n\right)\right) \\
& =h^{-1 / 2} r_{n} O_{P}\left(r_{n}^{2} \log n\right) \\
& =O_{P}\left(r_{n}^{3} h^{-1 / 2} \log n\right)
\end{aligned}
$$

Thus, $\left|I_{1}\right|=O_{P}\left(r_{n}^{3} h^{-1 / 2} \log n\right)$.

Next we study $I_{2}$. By an argument similar to (S.13), it can be shown that

$$
\frac{1}{n}\left\|\sum_{i=1}^{n} \ddot{\ell}_{a}\left(Y_{i} ; g_{0}\left(Z_{i}\right)\right) g\left(Z_{i}\right) K_{Z_{i}}-E\left\{\ddot{\ell}_{a}\left(Y_{i} ; g_{0}(Z)\right) g(Z) K_{Z}\right\}\right\|=O_{P}\left(a_{n}^{\prime} \log n\right) .
$$

Thus, $\left|I_{2}\right|=O_{P}\left(a_{n}^{\prime} r_{n} \log n\right)$.

Note $I_{3}=-\|g\|^{2} / 2$. Therefore, combining the above approximations of $I_{1}$ and $I_{2}$, we have $-2 n \cdot L R T_{n, \lambda}=n\left\|w_{0}+\widehat{g}^{0}-\widehat{g}\right\|^{2}+O_{P}\left(n r_{n} a_{n}^{\prime} \log n+n r_{n}^{3} h^{-1 / 2} \log n\right)=n\left\|w_{0}+\widehat{g}^{0}-\widehat{g}\right\|^{2}+$ $O_{P}\left(n r_{n} a_{n} \log n+n r_{n}^{3} h^{-1 / 2} \log n\right)$. By $r_{n}^{2} h^{-1 / 2}=o\left(a_{n}\right)$ and $n r_{n} a_{n}=o\left((\log n)^{-1}\right)$, it is easy to see that $O_{P}\left(n r_{n} a_{n} \log n+n r_{n}^{3} h^{-1 / 2} \log n\right)=o_{P}(1)$. Thus, part (ii) holds. So, to find the limiting distribution of the LRT test, we only focus on $n\left\|w_{0}+\widehat{g}^{0}-\widehat{g}\right\|^{2}$. By Theorems 3.4 and 4.3,

$$
n^{1 / 2}\left\|w_{0}+\widehat{g}^{0}-\widehat{g}-S_{n, \lambda}^{0}\left(g_{0}^{0}\right)+S_{n, \lambda}\left(g_{0}\right)\right\|=O_{P}\left(n^{1 / 2} a_{n} \log n\right)=o_{P}(1)
$$

so we just have to focus on $n^{1 / 2}\left\{S_{n, \lambda}^{0}\left(g_{0}^{0}\right)-S_{n, \lambda}\left(g_{0}\right)\right\}$. Recall that

$$
\begin{aligned}
S_{n, \lambda}^{0}\left(g_{0}^{0}\right) & =\frac{1}{n} \sum_{i=1}^{n} \epsilon_{i} K_{Z_{i}}^{*}-W_{\lambda}^{*} g_{0}^{0} \\
& =\frac{1}{n} \sum_{i=1}^{n} \epsilon_{i}\left(K_{Z_{i}}-K_{Z_{i}}\left(z_{0}\right) K_{z_{0}} / K\left(z_{0}, z_{0}\right)\right)-W_{\lambda} g_{0}+\left(W_{\lambda} g_{0}\right)\left(z_{0}\right) K_{z_{0}} / K\left(z_{0}, z_{0}\right),
\end{aligned}
$$

where $\epsilon_{i}=\dot{\ell}_{a}\left(Y_{i} ; g_{0}\left(Z_{i}\right)\right)$, and $S_{n, \lambda}\left(g_{0}\right)=\frac{1}{n} \sum_{i=1}^{n} \epsilon_{i} K_{Z_{i}}-W_{\lambda} g_{0}$. Thus,

$$
S_{n, \lambda}^{0}\left(g_{0}^{0}\right)-S_{n, \lambda}\left(g_{0}\right)=\left(-\frac{1}{n} \sum_{i=1}^{n} \epsilon_{i} K_{Z_{i}}\left(z_{0}\right)+\left(W_{\lambda} g_{0}\right)\left(z_{0}\right)\right) K_{z_{0}} / K\left(z_{0}, z_{0}\right) .
$$

So $n\left\|S_{n, \lambda}^{0}\left(g_{0}^{0}\right)-S_{n, \lambda}\left(g_{0}\right)\right\|^{2}=\left|\frac{1}{\sqrt{n}} \sum_{i=1}^{n} \epsilon_{i} K_{Z_{i}}\left(z_{0}\right) / \sqrt{K\left(z_{0}, z_{0}\right)}-\sqrt{n}\left(W_{\lambda} g_{0}\right)\left(z_{0}\right) / \sqrt{K\left(z_{0}, z_{0}\right)}\right|^{2}$. By central limit theorem, (4.10) and $\sqrt{n}\left(W_{\lambda} g_{0}\right)\left(z_{0}\right) / \sqrt{K\left(z_{0}, z_{0}\right)} \rightarrow-c_{z_{0}}$, we have

$$
\frac{1}{\sqrt{n}} \sum_{i=1}^{n} \epsilon_{i} K_{Z_{i}}\left(z_{0}\right) / \sqrt{K\left(z_{0}, z_{0}\right)}-\sqrt{n}\left(W_{\lambda} g_{0}\right)\left(z_{0}\right) / \sqrt{K\left(z_{0}, z_{0}\right)} \stackrel{d}{\longrightarrow} N\left(c_{z_{0}}, c_{0}\right) \text {. }
$$

It follows by (S.26)-(S.28) that $-2 n \cdot L R T_{n, \lambda} \stackrel{d}{\longrightarrow} c_{0} \chi_{1}^{2}\left(c_{z_{0}}^{2} / c_{0}\right)$, the scaled noncentral $\chi^{2}$ distribution with one degree of freedom and noncentrality parameter $c_{z_{0}}^{2} / c_{0}$, which shows (iii). It follows immediately that $\left\|w_{0}+\widehat{g}^{0}-\widehat{g}\right\|=O_{P}\left(n^{-1 / 2}\right)$, i.e., part (i) holds. This completes the proof. 
S.12. Proof of Corollary 4.5. By Fourier expansion of $g_{0}$ and $W_{\lambda} h_{\nu}=\frac{\lambda \gamma_{\nu}}{1+\lambda \gamma_{\nu}}$, we have $\left(W_{\lambda} g_{0}\right)\left(z_{0}\right)=$ $\sum_{\nu} V\left(g_{0}, h_{\nu}\right) \frac{\lambda \gamma_{\nu}}{1+\lambda \gamma_{\nu}} h_{\nu}\left(z_{0}\right)$. By the assumption that $\sum_{\nu}\left|V\left(g_{0}, h_{\nu}\right)\right|^{2} \gamma_{\nu}^{d}<\infty$, one obtains the bound $\left|\left(W_{\lambda} g_{0}\right)\left(z_{0}\right)\right|=O\left(\left(\lambda^{d} h^{-1}\right)^{1 / 2}\right)=O\left(h^{m d-1 / 2}\right)$ by using Cauchy's inequality. Thus, by $h \asymp n^{-1 /(2 m+1)}$ and $d>1+1 /(2 m),(n h)^{1 / 2}\left(W_{\lambda} g_{0}\right)\left(z_{0}\right)=o(1)$. Direct calculations verify $h=o(1), n h^{2} \rightarrow \infty$, $a_{n}=o\left((n h)^{-1 / 2}+h^{m}\right), a_{n}=o\left(n^{-1 / 2}(\log n)^{-1}\right), a_{n}=o\left(n^{-1}\left((n h)^{-1 / 2}+h^{m}\right)^{-1}(\log n)^{-1}\right)$, and $a_{n} \gg\left((n h)^{-1 / 2}+h^{m}\right)^{2} h^{-1 / 2}$. Thus, the desired result follows from Theorem 4.3.

S.13. Proof of Theorem 5.1. By Theorem 3.4 and Lemma 3.1,

$$
\left\|\widehat{g}-g_{0}^{*}-\frac{1}{n} \sum_{i=1}^{n} \epsilon_{i} K_{Z_{i}}\right\|_{\text {sup }}=O_{P}\left(a_{n} h^{-1 / 2} \log n\right) .
$$

So the key is to study the leading process $H_{n}(z)=n^{-1 / 2} \sum_{i=1}^{n} \epsilon_{i} K_{Z_{i}}(z)$.

Since $E\left\{\exp \left(|\epsilon| / C_{1}\right) \mid Z\right\} \leq C_{2}$, a.s., we may fix a sufficiently large constant $C>(1+3 \delta) C_{1}$ such that the event $E_{n}=\left\{\max _{1 \leq i \leq n}\left|\epsilon_{i}\right| \leq b_{n}=C \log n\right\}$ has large probability. Define $H_{n}^{b}(z)=$ $n^{-1 / 2} \sum_{i=1}^{n} \epsilon_{i} I\left(\left|\epsilon_{i}\right| \leq b_{n}\right) K_{Z_{i}}(z)$. Write $H_{n}(z)=H_{n}(z)-H_{n}^{b}(z)-E\left\{H_{n}(z)-H_{n}^{b}(z)\right\}+H_{n}^{b}(z)-$ $E\left\{H_{n}^{b}(z)\right\}$. Obviously, $H_{n}(z)-H_{n}^{b}(z)=0$ on $E_{n}$. By Chebyshev's inequality and Lemma 3.1, we have

$$
\begin{aligned}
\left|E\left\{H_{n}(z)-H_{n}^{b}(z)\right\}\right| & \left.=n^{1 / 2} \mid E\left\{\epsilon I\left(|\epsilon| \geq b_{n}\right) K_{Z}(z)\right)\right\} \mid \\
& \leq O(1) h^{-1 / 2} n^{1 / 2} E\left\{|\epsilon| \cdot I\left(|\epsilon| \geq b_{n}\right)\right\} \\
& \leq O(1) h^{-1 / 2} n^{1 / 2} E\left\{|\epsilon|^{2}\right\}^{1 / 2} P\left(|\epsilon|>b_{n}\right)^{1 / 2} \\
& =O\left(h^{-1 / 2} n^{1 / 2} \exp \left(-b_{n} /\left(2 C_{1}\right)\right)\right) .
\end{aligned}
$$

Thus,

$$
\sup _{z \in \mathbb{I}}\left|H_{n}(z)-H_{n}^{b}(z)-E\left\{H_{n}(z)-H_{n}^{b}(z)\right\}\right|=O_{P}\left(h^{-1 / 2} n^{1 / 2} \exp \left(-b_{n} /\left(2 C_{1}\right)\right)\right) .
$$

Denote $R_{n}(z)=H_{n}^{b}(z)-E\left\{H_{n}^{b}(z)\right\}$. Then by (S.30), we have

$$
\sup _{z \in \mathbb{I}}\left|H_{n}(z)-R_{n}(z)\right|=O_{P}\left(h^{-1 / 2} n^{1 / 2} \exp \left(-b_{n} /\left(2 C_{1}\right)\right)\right) .
$$

Let $Z_{n}(\epsilon, z)=n^{1 / 2}\left(P_{n}(\epsilon, z)-P(\epsilon, z)\right)$, where $P_{n}(\epsilon, z)$ and $P(\epsilon, z)$ are empirical and population distribution of $(\epsilon, Z)$. Then by Theorem 1 of [48], $\sup _{\epsilon \in \mathbb{R}, z \in \mathbb{I}}\left|Z_{n}(\epsilon, z)-W(\tau(\epsilon, z))\right|=O_{P}\left(n^{-1 / 2}(\log n)^{2}\right)$, where $W$ is Brownian bridge indexed by $[0,1] \times[0,1], \tau(\epsilon, z)=\left(P_{Z}(z), P_{\epsilon \mid Z}(\epsilon \mid z)\right), P_{Z}$ is the marginal distribution of $Z$, and $P_{\epsilon \mid Z}$ is the conditional distribution of $\epsilon$ given $Z$. Write

$$
\begin{gathered}
R_{n}^{b}(z)=\int_{0}^{1} \int_{-b_{n}}^{b_{n}} \epsilon K(z, t) d Z_{n}(\epsilon, t)=\int_{0}^{1} K(z, t) d V_{n}(t), \text { and } \\
R_{n}^{0}(z)=\int_{0}^{1} \int_{-b_{n}}^{b_{n}} \epsilon K(z, t) d W(\tau(\epsilon, t))=\int_{0}^{1} K(z, t) d V_{n}^{0}(t),
\end{gathered}
$$


where $V_{n}(t)=\int_{-b_{n}}^{b_{n}} \epsilon d Z_{n}(\epsilon, t)$ and $V_{n}^{0}(t)=\int_{-b_{n}}^{b_{n}} \epsilon d W(\tau(\epsilon, t))$. By integration by parts,

$$
\begin{aligned}
& V_{n}(t)=\left.Z_{n}(\epsilon, t) \epsilon\right|_{-b_{n}} ^{b_{n}}-\int_{-b_{n}}^{b_{n}} Z_{n}(\epsilon, t) d \epsilon, \text { and } \\
& V_{n}^{0}(t)=\left.W(\tau(\epsilon, t)) \epsilon\right|_{-b_{n}} ^{b_{n}}-\int_{-b_{n}}^{b_{n}} W(\tau(\epsilon, t)) d \epsilon .
\end{aligned}
$$

So $\sup _{t \in \mathbb{I}}\left|V_{n}(t)-V_{n}^{0}(t)\right|=O_{P}\left(b_{n} n^{-1 / 2}(\log n)^{2}\right)$.

By integration by parts again, we have

$$
\begin{gathered}
R_{n}(z)=\left.V_{n}(t) K(z, t)\right|_{t=0} ^{1}-\int_{0}^{1} V_{n}(t) \frac{d}{d t} K(z, t) d t, \text { and } \\
R_{n}^{0}(z)=\left.V_{n}^{0}(t) K(z, t)\right|_{t=0} ^{1}-\int_{0}^{1} V_{n}^{0}(t) \frac{d}{d t} K(z, t) d t .
\end{gathered}
$$

Therefore, by assumption $\sup _{z, t}\left|\frac{d}{d t} K(z, t)\right|=O\left(h^{-2}\right)$, we have

$$
\sup _{z \in \mathbb{I}}\left|R_{n}(z)-R_{n}^{0}(z)\right|=O_{P}\left(h^{-2} b_{n} n^{-1 / 2}(\log n)^{2}\right) .
$$

Write $W\left(t_{1}, t_{2}\right)=B\left(t_{1}, t_{2}\right)-t_{1} t_{2} B(1,1)$, where $B$ is standard Brownian motion indexed on $[0,1] \times[0,1]$. Define $\bar{R}_{n}^{0}(z)=\int_{0}^{1} K(z, t) d \bar{U}_{n}^{0}(t)$, where $\bar{U}_{n}^{0}(t)=\int_{-b_{n}}^{b_{n}} \epsilon d B(\tau(\epsilon, t))$. Direct calculations lead to $R_{n}^{0}(z)-\bar{R}_{n}^{0}(z)=B(1,1) \int_{0}^{1} K(z, t) \int_{-b_{n}}^{b_{n}} \epsilon d P(\epsilon, t)$. Therefore, by Lemma 3.1 and the finite exponential moment of $|\epsilon|$, we have

$$
\begin{aligned}
\sup _{z \in \mathbb{I}}\left|R_{n}^{0}(z)-\bar{R}_{n}^{0}(z)\right| & =|B(1,1)| \cdot \sup _{z \in \mathbb{I}}\left|\int_{0}^{1} K(z, t) \int_{-b_{n}}^{b_{n}} \epsilon d P_{\epsilon \mid Z}(\epsilon \mid t) d P_{Z}(t)\right| \\
& =|B(1,1)| \cdot \sup _{z \in \mathbb{I}}\left|\int_{0}^{1} K(z, t) E\left\{\epsilon I\left(|\epsilon| \leq b_{n}\right) \mid Z=t\right\} d P_{Z}(t)\right| \\
& =|B(1,1)| \cdot \sup _{z \in \mathbb{I}}\left|\int_{0}^{1} K(z, t) E\left\{\epsilon I\left(|\epsilon|>b_{n}\right) \mid Z=t\right\} d P_{Z}(t)\right| \\
& \leq c_{m}^{2} h^{-1}|B(1,1)| E\left\{|\epsilon| I\left(|\epsilon|>b_{n}\right)\right\} \\
& =O_{P}\left(h^{-1} \exp \left(-b_{n} /\left(2 C_{1}\right)\right)\right) .
\end{aligned}
$$

Define $\tilde{R}_{n}^{0}(z)=\int_{0}^{1} h^{-1} \omega((z-t) / h) d \bar{U}_{n}^{0}(t)$. Using integration by parts, we get $\bar{U}_{n}^{0}(t)=\left.B(\tau(\epsilon, t)) \epsilon\right|_{\epsilon=-b_{n}} ^{b_{n}}-$ $\int_{-b_{n}}^{b_{n}} B(\tau(\epsilon, t)) d \epsilon$, so we have $\sup _{t \in \mathbb{I}}\left|\bar{U}_{n}^{0}(t)\right|=O_{P}\left(b_{n}\right)$. Again, by integration by parts, we have $\bar{R}_{n}^{0}(z)-\tilde{R}_{n}^{0}(z)=\left.\bar{U}_{n}^{0}(t)\left(h^{-1} \omega((z-t) / h)-K(z, t)\right)\right|_{t=0} ^{1}-\int_{0}^{1} \bar{U}_{n}^{0}(t) \frac{d}{d t}\left(h^{-1} \omega((z-t) / h)-K(z, t)\right) d t$ which, by assumption (5.1), leads to

$$
\sup _{h^{\varphi} \leq z \leq 1-h^{\varphi}}\left|\bar{R}_{n}^{0}(z)-\tilde{R}_{n}^{0}(z)\right|=O_{P}\left(h^{-2} b_{n} \exp \left(-C_{2} h^{-1+\varphi}\right)\right) .
$$


By the proof of Lemma 3.7 in [20], the process $\tilde{R}_{n}^{0}(z)$ is Gaussian with mean zero and has the same distribution as the process $Y_{z}^{(n)}=h^{-1} \int_{0}^{1}\left(I_{n}(t)\right)^{1 / 2} \omega((z-t) / h) d W(t)$, where $W$ is standard one-dimensional Brownian motion indexed on $\mathbb{R}$ and $I_{n}(z)=E\left\{\epsilon^{2} I\left(|\epsilon| \leq b_{n}\right) \mid Z=z\right\}$. Define $Y_{0, z}^{(n)}=h^{-1} \int_{0}^{1}(I(t))^{1 / 2} \omega((z-t) / h) d W(t)$. Obviously, $\sup _{z \in \mathbb{I}}\left|I(z)-I_{n}(z)\right|=O\left(\exp \left(-b_{n} /\left(2 C_{1}\right)\right)\right)$. It follows from the assumption (5.5) and $E\left\{\exp \left(|\epsilon| / C_{1}\right) \mid Z\right\} \leq C$, a.s., that

$$
\begin{aligned}
\sup _{z \in \mathbb{I}}\left|\frac{d}{d z}\left(I(z)-I_{n}(z)\right)\right| & =\sup _{z \in \mathbb{I}}\left|\int_{|\epsilon|>b_{n}} \epsilon^{2} \frac{d}{d z} \pi(\epsilon \mid z) d \epsilon\right| \\
& \leq \sup _{z \in \mathbb{I}} \int_{|\epsilon|>b_{n}} \epsilon^{2} \rho_{1}\left(1+|\epsilon|^{\rho_{2}}\right) \pi(\epsilon \mid z) d \epsilon \\
& =\sup _{z \in \mathbb{I}} \rho_{1} E\left\{\epsilon^{2}\left(1+|\epsilon|^{\rho_{2}}\right) I\left(|\epsilon|>b_{n}\right) \mid Z=z\right\}=O\left(\exp \left(-b_{n} /\left(2 C_{1}\right)\right)\right) .
\end{aligned}
$$

By (5.5) and trivial calculations, it can be shown that $\sup _{t \in \mathbb{I}}\left|\frac{d}{d t} I(t)\right|<\infty$. Since, when $n$ is large, both $I$ and $I_{n}$ are bounded below from zero, we have

$$
\begin{aligned}
\left|\frac{d}{d t}\left(I(t)^{1 / 2}-I_{n}(t)^{1 / 2}\right)\right| & =(1 / 2)\left|\frac{I(t)^{\prime} I_{n}(t)^{1 / 2}-I_{n}(t)^{\prime} I(t)^{1 / 2}}{I(t)^{1 / 2} I_{n}(t)^{1 / 2}}\right| \\
& \leq(1 / 2) \frac{\left|I(t)^{\prime}\right| \cdot\left|I(t)^{1 / 2}-I_{n}(t)^{1 / 2}\right|+I(t)^{1 / 2}\left|I(t)^{\prime}-I_{n}(t)^{\prime}\right|}{I(t)^{1 / 2} I_{n}(t)^{1 / 2}} \\
& =O\left(\exp \left(-b_{n} /\left(2 C_{1}\right)\right)\right),
\end{aligned}
$$

where for convenience we denote $I^{\prime}(t)$ to be the derivative of $I(t)$. By integration by parts,

$$
\begin{aligned}
Y_{0, z}^{(n)}-Y_{z}^{(n)}= & \left.h^{-1} W(t)\left(I(t)^{1 / 2}-I_{n}(t)^{1 / 2}\right) \omega((z-t) / h)\right|_{t=0} ^{1} \\
& -h^{-1} \int_{0}^{1} W(t) \frac{d}{d t}\left(\left(I(t)^{1 / 2}-I_{n}(t)^{1 / 2}\right) \omega((z-t) / h)\right) d t \\
= & \left.h^{-1} W(t)\left(I(t)^{1 / 2}-I_{n}(t)^{1 / 2}\right) \omega((z-t) / h)\right|_{t=0} ^{1} \\
& -h^{-1} \int_{0}^{1} W(t) \frac{d}{d t}\left(I(t)^{1 / 2}-I_{n}(t)^{1 / 2}\right) \cdot \omega((z-t) / h) \\
& +h^{-2} \int_{0}^{1} W(t)\left(I(t)^{1 / 2}-I_{n}(t)^{1 / 2}\right) \cdot \omega^{\prime}((z-t) / h) d t
\end{aligned}
$$

for which we have

$$
\sup _{z \in \mathbb{Z}}\left|Y_{0, z}^{(n)}-Y_{z}^{(n)}\right|=O_{P}\left(h^{-2} \exp \left(-b_{n} /\left(2 C_{1}\right)\right)\right)
$$


Next we define $\bar{Y}_{0, z}^{(n)}=h^{-1} I(z)^{1 / 2} \int_{0}^{1} \omega((z-t) / h) d W(t)$. Then we have

$$
\begin{aligned}
Y_{0, z}^{(n)}-\bar{Y}_{0, z}^{(n)}= & h^{-1} \int_{0}^{1}\left(I(t)^{1 / 2}-I(z)^{1 / 2}\right) \omega((z-t) / h) d W(t) \\
= & h^{-1} \int_{z / h}^{(z-1) / h}\left(I(z-s h)^{1 / 2}-I(z)^{1 / 2}\right) \omega(s) d W(z-s h) \\
= & \left.h^{-1} W(z-s h)\left(I(z-s h)^{1 / 2}-I(z)^{1 / 2}\right) \omega(s)\right|_{s=z / h} ^{(z-1) / h} \\
& -h^{-1} \int_{z / h}^{(z-1) / h} W(z-s h) \frac{d}{d s}\left(\left(I(z-s h)^{1 / 2}-I(z)^{1 / 2}\right) \omega(s)\right) d s .
\end{aligned}
$$

Based on the facts that $\left|I(z-s h)^{1 / 2}-I(z)^{1 / 2}\right| \leq C_{I}|s| h$ for some positive constant $C_{I}$ and any $z, s \in \mathbb{I}$, that $|\omega(s)| \leq C_{\omega} \exp \left(-|s| / C_{3}\right)$ implying $|\omega(z / h)| \leq C_{\omega} \exp \left(-h^{\varphi-1} / C_{3}\right)=O(h)$ and $|\omega((z-1) / h)| \leq C_{\omega} \exp \left(-h^{\varphi-1} / C_{3}\right)=O(h)$ for $h^{\varphi} \leq z \leq 1-h^{\varphi}$, and that $\omega^{\prime}$ is bounded, it can be verified that

$$
\sup _{h^{\varphi} \leq z \leq 1-h^{\varphi}}\left|Y_{0, z}^{(n)}-\bar{Y}_{0, z}^{(n)}\right|=O_{P}(1)
$$

The last random process we will consider is $L_{z}^{(n)}=h^{-1} I(z)^{1 / 2} \int_{\mathbb{R}} \omega((z-t) / h) d W(t)$. We will establish the rate of convergence for $\sup _{h^{\varphi} \leq z \leq 1-h^{\varphi}}\left|L_{z}^{(n)}-\bar{Y}_{0, z}^{(n)}\right|$. For this purpose, we need the following result.

Lemma S.4. For any $\kappa>1 / 2, \lim _{d \rightarrow \infty} P\left(\sup _{s \in \mathbb{R}} \frac{|W(s)|}{(1+|s|)^{\kappa}}>d\right)=0$.

Proof of Lemma S.4. Let $D_{\kappa}=\sup _{s>0} \frac{|W(s)|}{(1+s)^{\kappa}}$. We will only show $\lim _{d \rightarrow \infty} P\left(D_{\kappa}>d\right)=0$. The proof for $\sup _{s \leq 0} \frac{|W(s)|}{(1+s)^{\kappa}}$ is similar. Let $\mathbb{Z}_{+}=\{0,1, \ldots\}$ be the set of nonnegative integers. Note $\sup _{s>0} \frac{|W(s)|}{(1+s)^{\kappa}}=\sup _{m \in \mathbb{Z}_{+}} \sup _{m<s \leq m+1} \frac{|W(s)|}{(1+s)^{\kappa}}$. Choose a constant $\beta>0$ such that $(\beta+1)(\kappa-1 / 2)>$ 1. Then

$$
\begin{aligned}
P\left(D_{\kappa}>d\right) & =P\left(\sup _{m \in \mathbb{Z}_{+}} \sup _{m<s \leq m+1} \frac{|W(s)|}{(1+s)^{\kappa}}>d\right) \\
& \leq \sum_{m=0}^{\infty} P\left(\sup _{m<s \leq m+1} \frac{|W(s)|}{(1+s)^{\kappa}}>d\right) \\
& \leq \sum_{m=0}^{\infty} P\left(\sup _{m<s \leq m+1}|W(s)|>(1+m)^{\kappa} d\right) \\
& \leq \sum_{m=0}^{\infty} P\left(\sup _{0<s \leq m+1}|W(s)|>(1+m)^{\kappa} d\right) \\
& \leq \frac{4}{(2 \pi)^{1 / 2}} \sum_{m=0}^{\infty} \frac{\exp \left(-\left(d(1+m)^{\kappa-1 / 2}\right)^{2} / 2\right)}{d(1+m)^{\kappa-1 / 2}} \\
& \leq \frac{4}{(2 \pi)^{1 / 2}} \sum_{m=0}^{\infty} \frac{1}{\left(d(1+m)^{\kappa-1 / 2}\right)^{\beta+1}}=O\left(d^{-(\beta+1)}\right)
\end{aligned}
$$


where (S.37) follows by [26]. Therefore, the desired result holds.

Now define $E_{n, 1}=\left\{\sup _{s \in \mathbb{R}} \frac{|W(s)|}{(1+|s|)^{\kappa}} \leq d\right\}$ for some fixed $d>0$ so that $E_{n, 1}$ has large probability. By integration by parts and a straightforward calculation, we have

$$
\begin{aligned}
L_{z}^{(n)}-\bar{Y}_{0, z}^{(n)}= & h^{-1} I(z)^{1 / 2}\left(\int_{-\infty}^{0} \omega((z-t) / h) d W(t)+\int_{1}^{\infty} \omega((z-t) / h) d W(t)\right) \\
= & h^{-1} I(z)^{1 / 2}\left(\left.W(t) \omega((z-t) / h)\right|_{t=-\infty} ^{0}-\int_{-\infty}^{0} W(t) \omega^{\prime}((z-t) / h) h^{-1} d t\right) \\
& +h^{-1} I(z)^{1 / 2}\left(\left.W(t) \omega((z-t) / h)\right|_{t=1} ^{\infty}-\int_{1}^{\infty} W(t) \omega^{\prime}((z-t) / h) h^{-1} d t\right) .
\end{aligned}
$$

On $E_{1, n}$, for any $z$, we have $|W(z)| \leq d(1+|z|)^{\kappa}$. By assumption $\left.(5.1), \mid \omega((z-t) / h)\right) \mid \leq$ $\left.C_{\omega} \exp \left(-|z-t| /\left(h C_{3}\right)\right), \mid \omega^{\prime}((z-t) / h)\right) \mid \leq C_{\omega} \exp \left(-|z-t| /\left(h C_{3}\right)\right)$. Thus we have, for any fixed $z$, as $|t| \rightarrow \infty$

$$
|W(t) \omega((z-t) / h)| \leq d C_{\omega}(1+|t|)^{\kappa} \exp \left(-|z-t| /\left(h C_{3}\right)\right) \longrightarrow 0 .
$$

Meanwhile, on $E_{1, n}$, we have $|W(1) \omega((z-1) / h)| \leq 2 d \exp \left(-h^{\varphi-1} / C_{3}\right)$, and

$$
\begin{aligned}
\left|\int_{1}^{\infty} W(t) \omega^{\prime}((z-t) / h) d t\right| & \leq \int_{1}^{\infty} d(1+t)^{\kappa} \cdot C_{\omega} \exp \left(-|z-t| /\left(C_{3} h\right)\right) d t \\
& =\int_{1}^{\infty} d(1+t)^{\kappa} \cdot C_{\omega} \exp \left(-(t-z) /\left(C_{3} h\right)\right) d t \\
& =\int_{1-z}^{\infty} d(1+t+z)^{\kappa} \cdot C_{\omega} \exp \left(-t /\left(C_{3} h\right)\right) d t \\
& \leq \int_{h^{\varphi}}^{\infty} d(2+t)^{\kappa} \cdot C_{\omega} \exp \left(-t /\left(C_{3} h\right)\right) d t \\
& =h^{\varphi} \int_{1}^{\infty} d\left(2+h^{\varphi} t\right)^{\kappa} \cdot C_{\omega} \exp \left(-t /\left(C_{3} h^{1-\varphi}\right)\right) d t \\
& \leq h^{\varphi} \int_{1}^{\infty} d(2+t)^{\kappa} \cdot C_{\omega}\left(t /\left(C_{3} h^{1-\varphi}\right)\right)^{-a} d t \\
& =C_{3}^{a} d C_{\omega} h^{\varphi+a(1-\varphi)} \int_{1}^{\infty}(2+t)^{\kappa} t^{-a} d t=O\left(h^{\varphi+a(1-\varphi)}\right)=O\left(h^{3}\right),
\end{aligned}
$$

where $a$ is constant with $a>\kappa+2$ and $\varphi+a(1-\varphi)>3$. Using a similar technique, one can show that on $E_{1, n},\left|\int_{-\infty}^{0} W(t) \omega^{\prime}((z-t) / h) d t\right| \leq O\left(h \exp \left(-h^{\varphi-1} / C_{3}\right)\right)$. Consequently,

$$
\sup _{h^{\varphi} \leq z \leq 1-h^{\varphi}}\left|L_{z}^{(n)}-\bar{Y}_{0, z}^{(n)}\right|=O_{P}(h)
$$

Since $h^{1 / 2} L_{z}^{(n)} I(z)^{-1 / 2} / \sigma_{\omega}=h^{-1 / 2} \int \omega((t-z) / h) d W(t) / \sigma_{\omega}$ is stationary Gaussian with mean zero, the process $h^{1 / 2} L_{h z}^{(n)} I(h z)^{-1 / 2} / \sigma_{\omega}$ is Gaussian with mean zero and covariance function 
$\int_{-\infty}^{\infty} \omega(t) \omega(t+\cdot) d t / \sigma_{\omega}^{2}$. Then by [4], we have as $n \rightarrow \infty$,

$$
\begin{aligned}
& P\left((2 \delta \log n)^{1 / 2}\left\{\sup _{h \varphi \leq z \leq 1-h^{\varphi}}\left|h^{1 / 2} L_{z}^{(n)} I(z)^{-1 / 2} \sigma_{\omega}^{-1}\right|-d_{n}\right\} \leq u\right) \\
= & P\left((2 \delta \log n)^{1 / 2}\left\{\sup _{0 \leq z \leq 1-2 h^{\varphi}}\left|h^{1 / 2} L_{z}^{(n)} I(z)^{-1 / 2} \sigma_{\omega}^{-1}\right|-d_{n}\right\} \leq u\right) \\
= & P\left((2 \delta \log n)^{1 / 2}\left\{\sup _{0 \leq z \leq h^{-1}\left(1-2 h^{\varphi}\right)}\left|h^{1 / 2} L_{h z}^{(n)} I(h z)^{-1 / 2} \sigma_{\omega}^{-1}\right|-d_{n}\right\} \leq u\right) \\
\rightarrow & \exp (-\exp (-2 u)),
\end{aligned}
$$

where $\sigma_{\omega}=\left(\int_{\mathbb{R}} \omega(u)^{2} d u\right)^{1 / 2}$. By assumption $C>(3 \delta+1) C_{1}, m>(3+\sqrt{5}) / 4$ and $0<\delta<$ $2 m /(8 m-1)$, the remainders in (S.29), (S.31)-(S.38) are all $o_{P}\left((h \log n)^{-1 / 2}\right)$. Thus the desired conclusion holds.

S.14. Proof of Proposition 5.2. We first consider (5.2) and (5.3). (5.2) trivially holds. By boundedness and absolute integrability of $\omega$, for any $\rho \in(0,2], \lim _{|z| \rightarrow \infty} \int_{-\infty}^{\infty} \frac{\omega(t)(\omega(t+z)-\omega(t))}{|z|^{\rho}} d t=$ 0 , implying $C_{\rho}$ in (5.3) is actually zero.

For general $m$, let $\tilde{h}_{\nu}$ and $\tilde{\gamma}_{\nu}$ be the normalized (with respect to the usual $L_{2}$-norm) eigenfunctions and eigenvalues of the boundary value problem $(-1)^{m} \tilde{h}_{\nu}^{(2 m)}=\tilde{\gamma}_{\nu} \tilde{h}_{\nu}, \widetilde{h}_{\nu}^{(j)}(0)=\widetilde{h}_{\nu}^{(j)}(1)=0$, $j=m, m+1, \ldots, 2 m-1$. Thus, it is easy to see that $h_{\nu}=\sigma \widetilde{h}_{\nu}$ and $\gamma_{\nu}=\sigma^{2} \widetilde{\gamma}_{\nu}$ satisfy (2.11) with $\pi(z) I(z) \equiv \sigma^{-2}$, implying that $h_{\nu}$ and $\gamma_{\nu}$ form an effective eigensystem in $\mathcal{H}$. Let $\lambda^{\dagger}=\sigma^{2} \lambda$ and $h^{\dagger}=\sigma^{1 / m} h$. Define $\widetilde{K}(s, t)=\sum_{\nu} \frac{\widetilde{h}_{\nu}(s) \widetilde{h}_{\nu}(t)}{1+\lambda^{\dagger} \widetilde{\gamma}_{\nu}}$. Then $\widetilde{K}$ is the reproducing kernel function associated with the inner product $\langle f, g\rangle_{1}=\int_{0}^{1} f(t) g(t) d t+\lambda^{\dagger} \int_{0}^{1} f^{(m)}(t) g^{(m)}(t) d t$. Thus, $\widetilde{K}$ is the Green's function associated with the differential equation (2.1) in [37], with the penalty parameter therein replaced by $\lambda^{\dagger}$.

Next we restrict $m=2$. By Theorem 4.1 in [34], for $j=0,1$, we have

$$
\sup _{s, t \in \mathbb{I}}\left|\frac{d^{j}}{d t^{j}}(\widetilde{K}(s, t)-\bar{K}(s, t))\right| \leq C_{K}^{\prime}\left(h^{\dagger}\right)^{-(j+1)} \exp \left(-\sin (\pi /(2 m)) / h^{\dagger}\right),
$$

where by equation (6) in [34], $\bar{K}$ satisfies for any $s, t \in \mathbb{I}$ and $j=0,1$,

$$
\left|\frac{d^{j}}{d t^{j}}\left(\bar{K}(s, t)-\frac{1}{h^{\dagger}} \omega_{0}\left(\frac{s-t}{h^{\dagger}}\right)\right)\right| \leq C_{K}^{\prime \prime}\left(h^{\dagger}\right)^{-(j+1)}\left(\exp \left(-|1-s| /\left(\sqrt{2} h^{\dagger}\right)\right)+\exp \left(-|s| /\left(\sqrt{2} h^{\dagger}\right)\right)\right),
$$

with $C_{K}^{\prime}, C_{K}^{\prime \prime}$ both being positive constants. By (S.40) and (S.41), it is easy to see that for any $s, t \in \mathbb{I}$ and $j=0,1$,

$$
\begin{aligned}
& \left|\frac{d^{j}}{d t^{j}}\left(\widetilde{K}(s, t)-\frac{1}{h^{\dagger}} \omega_{0}\left(\frac{s-t}{h^{\dagger}}\right)\right)\right| \\
\leq & C_{K}\left(h^{\dagger}\right)^{-(j+1)}\left(\exp \left(-\sin (\pi /(2 m)) / h^{\dagger}\right)+\exp \left(-|1-s| /\left(\sqrt{2} h^{\dagger}\right)\right)+\exp \left(-|s| /\left(\sqrt{2} h^{\dagger}\right)\right)\right),
\end{aligned}
$$


where $C^{\prime}, C_{K}$ are positive constant. By Proposition 2.1, $K(s, t)=\sum_{\nu} \frac{h_{\nu}(s) h_{\nu}(t)}{1+\lambda \gamma_{\nu}}=\sigma^{2} \widetilde{K}(s, t)$. Therefore, $K(s, t)-h^{-1} \omega((s-t) / h)=\sigma^{2}\left(\widetilde{K}(s, t)-\left(h^{\dagger}\right)^{-1} \omega_{0}\left((s-t) / h^{\dagger}\right)\right)$. It can thus be shown that, by (S.42), Condition (5.1) holds.

S.15. Proof of Theorem 5.3. For simplicity, denote $\widehat{g}=\widehat{g}_{n, \lambda}$ and $g=\widehat{g}-g_{0}$. Using arguments similar to (S.21), (S.22) and (S.25), and by assumption $a_{n}=o\left(r_{n}\right), n r_{n} a_{n} \log n=o\left(h^{-1 / 2}\right)$, $n r_{n}^{3} h^{-1 / 2} \log n=o\left(n r_{n} a_{n} \log n\right)=o\left(h^{-1 / 2}\right)$, it can be shown that

$$
-2 n \cdot P L R T_{n, \lambda}=n\left\|\widehat{g}-g_{0}\right\|^{2}+O_{P}\left(n r_{n} a_{n} \log n+n r_{n}^{3} h^{-1 / 2} \log n\right)=n\left\|\widehat{g}-g_{0}\right\|^{2}+o_{P}\left(h^{-1 / 2}\right) \text {. }
$$

Under the hypothesis $H_{0}^{\text {global }}$ that $g_{0}$ is the "true" parameter, by Theorem 3.4, we have $\| \widehat{g}-g_{0}-$ $S_{n, \lambda}\left(g_{0}\right) \|=O_{P}\left(a_{n} \log n\right)$, where $a_{n}$ is defined as in (3.5). It thus follows from $n^{1 / 2} a_{n} \log n=o(1)$ that $n^{1 / 2}\left\|\widehat{g}-g_{0}\right\|=n^{1 / 2}\left\|S_{n, \lambda}\left(g_{0}\right)\right\|+o_{P}(1)$.

Next we study the leading term $\left\|S_{n, \lambda}\left(g_{0}\right)\right\|$. We first approximate $\left\|W_{\lambda} g_{0}\right\|$. By Proposition 2.1 and the dominated convergence theorem, it can be established that

$$
\left\|W_{\lambda} g_{0}\right\|^{2}=o(\lambda)
$$

To see (S.44), define $f_{\lambda}(\nu)=\left|V\left(g_{0}, h_{\nu}\right)\right|^{2} \gamma_{\nu} \frac{\lambda \gamma_{\nu}}{1+\lambda \gamma_{\nu}}$, for $\nu=0,1, \ldots, \lambda>0$. Then $f_{\lambda}$ is a sequence of functions satisfying $\left|f_{\lambda}(\nu)\right| \leq\left|V\left(g_{0}, h_{\nu}\right)\right|^{2} \gamma_{\nu} \equiv f(\nu)$. From $g_{0} \in \mathcal{H}$, we have $\sum_{\nu \in \mathbb{N}}\left|V\left(g_{0}, h_{\nu}\right)\right|^{2} \gamma_{\nu}=$ $\int_{\mathbb{N}} f(\nu) d m(\nu)<\infty$, where $m(\cdot)$ denotes the discrete measure over $\mathbb{N}$. So $f(\nu)$ is an integrable function over $\mathbb{N}$ which dominates $f_{\lambda}(\nu)$. Since $\lim _{\lambda \rightarrow 0} f_{\lambda}(\nu)=0$, we have $\sum_{\nu}\left|V\left(g_{0}, h_{\nu}\right)\right|^{2} \frac{\lambda \gamma_{\nu}^{2}}{1+\lambda \gamma_{\nu}}=$ $\int_{\mathbb{N}} f_{\lambda}(\nu) d m(\nu) \rightarrow 0$ based on the Lebesgue dominated convergence theorem. That is, $\left\|W_{\lambda} g_{0}\right\|^{2}=$ $\sum_{\nu}\left|V\left(g_{0}, h_{\nu}\right)\right|^{2} \frac{\lambda^{2} \gamma_{\nu}^{2}}{1+\lambda \gamma_{\nu}}=o(\lambda)$.

By (2.12), $n\left\|S_{n, \lambda}\left(g_{0}\right)\right\|^{2}=n^{-1}\left\|\sum_{i=1}^{n} \epsilon_{i} K_{Z_{i}}\right\|^{2}-2 \sum_{i=1}^{n} \epsilon_{i}\left(W_{\lambda} g_{0}\right)\left(Z_{i}\right)+n\left\|W_{\lambda} g_{0}\right\|^{2}$. It follows by the Fourier expansion of $g_{0}$ and Proposition 2.1 that

$$
\begin{aligned}
& E\left\{\left|\sum_{i=1}^{n} \epsilon_{i}\left(W_{\lambda} g_{0}\right)\left(Z_{i}\right)\right|^{2}\right\} \\
= & n E\left\{\epsilon^{2}\left|\left(W_{\lambda} g_{0}\right)(Z)\right|^{2}\right\}=n V\left(W_{\lambda} g_{0}, W_{\lambda} g_{0}\right)=n \sum_{\nu}\left|V\left(g_{0}, h_{\nu}\right)\right|^{2}\left(\frac{\lambda \gamma_{\nu}}{1+\lambda \gamma_{\nu}}\right)^{2}=o(n \lambda),
\end{aligned}
$$

where the last equality follows by $\sum_{\nu}\left|V\left(g_{0}, h_{\nu}\right)\right|^{2} \gamma_{\nu}<\infty$ and the dominated convergence theorem; see (S.44) for similar arguments examining $\left\|W_{\lambda} g_{0}\right\|$. So $\sum_{i=1}^{n} \epsilon_{i}\left(W_{\lambda} g_{0}\right)\left(Z_{i}\right)=o_{P}\left((n \lambda)^{1 / 2}\right)=$ $o_{P}\left(h^{-1 / 2}\right)$. Thus, $n\left\|W_{\lambda} g_{0}\right\|^{2}=o(n \lambda)$. Consequently, $n\left\|S_{n, \lambda}\left(g_{0}\right)\right\|^{2}=n^{-1}\left\|\sum_{i=1}^{n} \epsilon_{i} K_{Z_{i}}\right\|^{2}+n\left\|W_{\lambda} g_{0}\right\|^{2}+$ $o_{P}\left(h^{-1 / 2}\right)=n^{-1}\left\|\sum_{i=1}^{n} \epsilon_{i} K_{Z_{i}}\right\|^{2}+o(n \lambda)+o_{P}\left(h^{-1 / 2}\right)$. In what follows, we study the limiting property of $n^{-1}\left\|\sum_{i=1}^{n} \epsilon_{i} K_{Z_{i}}\right\|^{2}$.

Write $n^{-1}\left\|\sum_{i=1}^{n} \epsilon_{i} K_{Z_{i}}\right\|^{2}=n^{-1} \sum_{i=1}^{n} \epsilon_{i}^{2} K\left(Z_{i}, Z_{i}\right)+n^{-1} W(n)$, where $W(n)=\sum_{i \neq j} \epsilon_{i} \epsilon_{j} K\left(Z_{i}, Z_{j}\right)$. If we denote $W_{i j}=2 \epsilon_{i} \epsilon_{j} K\left(Z_{i}, Z_{j}\right)$, then we can rewrite $W(n)$ as $\sum_{1 \leq i<j \leq n} W_{i j}$ so that $W(n)$ is 
clean (see [12]). Next we will derive the limiting distribution for $W(n)$. Let $\sigma(n)^{2}=\operatorname{Var}(W(n))$, and $G_{I}, G_{I I}, G_{I V}$ be defined as

$$
\begin{gathered}
G_{I}=\sum_{i<j} E\left\{W_{i j}^{4}\right\}, \\
G_{I I}=\sum_{i<j<k}\left(E\left\{W_{i j}^{2} W_{i k}^{2}\right\}+E\left\{W_{j i}^{2} W_{j k}^{2}\right\}+E\left\{W_{k i}^{2} W_{k j}^{2}\right\}\right), \text { and } \\
G_{I V}=\sum_{i<j<k<l}\left(E\left\{W_{i j} W_{i k} W_{l j} W_{l k}\right\}+E\left\{W_{i j} W_{i l} W_{k j} W_{k l}\right\}+E\left\{W_{i k} W_{i l} W_{j k} W_{j l}\right\}\right) .
\end{gathered}
$$

It follows from Proposition 3.2 of [12] that, to show $\sigma(n)^{-1} W(n) \stackrel{d}{\rightarrow} N(0,1)$, it is sufficient to show that $G_{I}, G_{I I}, G_{I V}$ are of lower order than $\sigma(n)^{4}$. By assumption $E\left\{\epsilon^{4} \mid Z\right\} \leq C$, a.s., we have $E\left\{\epsilon^{4} \mid Z\right\} \leq C \leq C C_{2} I(Z)$, a.s. It then follows from (S.5) that $E\left\{W_{i j}^{4}\right\}=16 E\left\{\epsilon_{i}^{4} \epsilon_{j}^{4} K\left(Z_{i}, Z_{j}\right)^{4}\right\}=$ $O\left(h^{-4}\right)$, which implies $G_{I}=O\left(n^{2} h^{-4}\right)$. Obviously, $E\left\{W_{i j}^{2} W_{i k}^{2}\right\} \leq E\left\{W_{i j}^{4}\right\}=O\left(h^{-4}\right)$, implying $G_{I I}=O\left(n^{3} h^{-4}\right)$.

To approximate $G_{I V}$, for pairwise different $i, j, k, l$, we have

$$
\begin{aligned}
E\left\{W_{i j} W_{i k} W_{l j} W_{l k}\right\} & =16 E\left\{\epsilon_{i}^{2} \epsilon_{j}^{2} \epsilon_{l}^{2} \epsilon_{k}^{2} K\left(Z_{i}, Z_{j}\right) K\left(Z_{i}, Z_{k}\right) K\left(Z_{l}, Z_{j}\right) K\left(Z_{l}, Z_{k}\right)\right\} \\
& =\sum_{\nu} \frac{1}{\left(1+\lambda \gamma_{\nu}\right)^{4}}=O\left(h^{-1}\right)
\end{aligned}
$$

based on the direct examination. Therefore, $G_{I V}=O\left(n^{4} h^{-1}\right)$.

Next we obtain the exact order of $\sigma(n)^{4}$, which is $n^{4} h^{-2}$. This follows from the observation $E\left\{W_{i j}^{2}\right\}=4 E\left\{\epsilon_{i}^{2} \epsilon_{j}^{2} K\left(Z_{i}, Z_{j}\right)^{2}\right\}=4 h^{-1} \rho_{K}^{2}$. Thus, $\sigma(n)^{4}=\left(\left(\begin{array}{c}n \\ 2\end{array}\right) E\left\{W_{i j}^{2}\right\}\right)^{2}$ has the same order as $4 n^{4} h^{-2} \rho_{K}^{4}$. It follows by $h=o(1)$ and $\left(n h^{2}\right)^{-1}=o(1)$ that $G_{I}, G_{I I}$ and $G_{I V}$ are of lower order than $\sigma(n)^{4}$, which implies by Proposition 3.2 of [12] that

$$
\frac{1}{\sqrt{2 h^{-1}} n \rho_{K}} W(n) \stackrel{d}{\rightarrow} N(0,1) .
$$

To conclude, we approximate the term $\sum_{i=1}^{n} \epsilon_{i}^{2} K\left(Z_{i}, Z_{i}\right)$. By $E\left\{\epsilon^{4} \mid Z\right\} \leq C$, a.s., we have $E\left\{\epsilon^{4} K(Z, Z)^{2}\right\}=$ $O\left(h^{-2}\right)$. Therefore, a direct calculation leads to $E\left\{\left|\sum_{i=1}^{n}\left[\epsilon_{i}^{2} K\left(Z_{i}, Z_{i}\right)-h^{-1} \sigma_{K}^{2}\right]\right|^{2}\right\} \leq n E\left\{\epsilon^{4} K(Z, Z)^{2}\right\}=$ $O\left(n h^{-2}\right)$, where $\sigma_{K}^{2}=h E\left\{\epsilon^{2} K(Z, Z)\right\}$. This implies $\sum_{i=1}^{n}\left[\epsilon_{i}^{2} K\left(Z_{i}, Z_{i}\right)-h^{-1} \sigma_{K}^{2}\right]=O_{P}\left(n^{1 / 2} h^{-1}\right)$.

Therefore,

$$
n^{-1} \sum_{i=1}^{n} \epsilon_{i}^{2} K\left(Z_{i}, Z_{i}\right)=h^{-1} \sigma_{K}^{2}+O_{P}\left(n^{-1 / 2} h^{-1}\right)=h^{-1} \sigma_{K}^{2}+O_{P}(1)
$$

From (S.45) and (S.46), $(h / n)\left\|\sum_{i=1}^{n} \epsilon_{i} K_{Z_{i}}\right\|^{2}=\sigma_{K}^{2}+o_{P}(1)$, which implies $n\left\|S_{n, \lambda}\left(g_{0}\right)\right\|^{2}=$ 
$O_{P}\left(h^{-1}+n \lambda+h^{-1 / 2}\right)=O_{P}\left(h^{-1}\right)$, and hence $n^{1 / 2}\left\|S_{n, \lambda}\left(g_{0}\right)\right\|=O_{P}\left(h^{-1 / 2}\right)$. Thus,

$$
\begin{aligned}
-2 n \cdot P L R T_{n, \lambda} & =n\left\|\widehat{g}-g_{0}\right\|^{2}+o_{P}\left(h^{-1 / 2}\right) \\
& =\left(n^{1 / 2}\left\|S_{n, \lambda}\left(g_{0}\right)\right\|+o_{P}(1)\right)^{2}+o_{P}\left(h^{-1 / 2}\right) \\
& =n\left\|S_{n, \lambda}\left(g_{0}\right)\right\|^{2}+2 n^{1 / 2}\left\|S_{n, \lambda}\left(g_{0}\right)\right\| \cdot o_{P}(1)+o_{P}\left(h^{-1 / 2}\right) \\
& =n^{-1}\left\|\sum_{i=1}^{n} \epsilon_{i} K_{Z_{i}}\right\|^{2}+n\left\|W_{\lambda} g_{0}\right\|^{2}+o_{P}\left(h^{-1 / 2}\right) .
\end{aligned}
$$

It follows by (S.45)-(S.47) and Slutsky's theorem that $\left(2 h^{-1} \sigma_{K}^{4} / \rho_{K}^{2}\right)^{-1 / 2}\left(-2 n r_{K} \cdot P L R T_{n, \lambda}-\right.$ $\left.n r_{K}\left\|W_{\lambda} g_{0}\right\|^{2}-h^{-1} \sigma_{K}^{4} / \rho_{K}^{2}\right) \stackrel{d}{\longrightarrow} N(0,1)$.

S.16. Proof of Theorem 5.4. First of all, by direct calculations, one can verify by $\frac{1}{2 m+1} \leq d<$ $\frac{2 m}{8 m-1}$ and $m>\frac{3+\sqrt{5}}{4}$ that $h \asymp n^{-d}$ satisfies the conditions in Theorem 5.3.

Next we prove our theorem. We write

$$
-2 n \cdot P L R T_{n, \lambda}=-2 n\left(\ell_{n, \lambda}\left(g_{0}\right)-\ell_{n, \lambda}\left(g_{n 0}\right)\right)-2 n\left(\ell_{n, \lambda}\left(g_{n 0}\right)-\ell_{n, \lambda}\left(\widehat{g}_{n, \lambda}\right)\right) .
$$

The proof proceeds in two parts. Firstly. We note that $-2 n \cdot P L R T^{\prime} \equiv-2 n\left(\ell_{n, \lambda}\left(g_{n 0}\right)-\ell_{n, \lambda}\left(\widehat{g}_{n, \lambda}\right)\right)$ is actually the PLRT test for testing $H_{1 n}$ against $H_{1}^{\text {global }}$. Under $H_{1 n},-2 n \cdot P L R T^{\prime}$ has the same asymptotic distribution as in Theorem 5.3, but uniformly for all $g_{n} \in \mathcal{G}_{a}$. That is to say, $\left(2 u_{n}\right)^{-1 / 2}\left(-2 n r_{K} \cdot P L R T^{\prime}-n\left\|W_{\lambda} g_{n 0}\right\|^{2}-u_{n}\right)=O_{P}(1)$ uniformly for $g_{n} \in \mathcal{G}_{a}$, where $u_{n}=h^{-1} \sigma_{K}^{4} / \rho_{K}^{2}$ with $\sigma_{K}^{2}$ and $\rho_{K}^{2}$ given in (5.14). Secondly, we show that $-2 n\left(\ell_{n, \lambda}\left(g_{0}\right)-\right.$ $\left.\ell_{n, \lambda}\left(g_{n 0}\right)\right)=n\left\|g_{n}\right\|^{2}+O_{P}\left(n^{1 / 2}\left\|g_{n}\right\|+n^{1 / 2}\left\|g_{n}\right\|^{2}+n \lambda\right)$. Then $\left(2 u_{n}\right)^{-1 / 2}\left(-2 n r_{K} \cdot P L R T-u_{n}\right) \geq$ $n\left(2 u_{n}\right)^{-1 / 2}\left\|g_{n}\right\|^{2}\left(1+O_{P}\left(n^{-1 / 2}\left\|g_{n}\right\|^{-1}+n^{-1 / 2}+\lambda\left\|g_{n}\right\|^{-2}\right)\right)+\left(2 u_{n}\right)^{-1 / 2} n\left\|W_{\lambda} g_{n 0}\right\|^{2}+O_{P}(1) \geq$ $n\left(2 u_{n}\right)^{-1 / 2}\left\|g_{n}\right\|^{2}\left(1+O_{P}\left(n^{-1 / 2}\left\|g_{n}\right\|^{-1}+n^{-1 / 2}+\lambda\left\|g_{n}\right\|^{-2}\right)\right)+O_{P}(1)$, where $O_{P}(\cdot)$ holds uniformly for $g_{n} \in \mathcal{G}_{a}$. Let $n^{-1 / 2}\left\|g_{n}\right\|^{-1} \leq 1 / C, \lambda\left\|g_{n}\right\|^{-2} \leq 1 / C$ and $\left\|g_{n}\right\|^{2} \geq C\left(n h^{1 / 2}\right)$ for sufficiently large $C$, which implies that $\left|\frac{-2 n r_{K} \cdot P L R T-u_{n}}{\left(2 u_{n}\right)^{1 / 2}}\right| \geq c_{\alpha}$ with large probability, where $c_{\alpha}$ is the cutoff value (based on $N(0,1)$ ) for rejecting $H_{0}^{\text {global }}$ at level $\alpha$. This means that we have to assume $\left\|g_{n}\right\|^{2} \geq C\left(\lambda+\left(n h^{1 / 2}\right)^{-1}\right)$ to achieve large power.

Next we complete the above two parts. Firstly, it can be established that the following "uniform" FBR holds, i.e., for any $\delta \in(0,1)$, there exist positive constants $\widetilde{C}$ and $N$ such that

$$
\inf _{n \geq N} \inf _{g_{n} \in \mathcal{G}_{a}} P_{g_{n 0}}\left(\left\|\widehat{g}_{n, \lambda}-g_{n 0}-S_{n, \lambda}\left(g_{n 0}\right)\right\| \leq \widetilde{C} a_{n}\right) \geq 1-\delta
$$

where $a_{n}$ is defined in (3.5), The proof of (S.49) follows by a careful reexamination of Theorem 3.4. Specifically, one can choose $C$ and $M$ (to be unrelated to $g_{n} \in \mathcal{G}_{a}$ ) to be large so that the event $B_{n 1} \cap B_{n 2}$, which is defined in the proof of Theorem 3.4, has probability greater than $1-\frac{\delta}{4}$. Then by going through exactly the same proof, it can be shown that when $n \geq N$ for some suitably selected $N$, (S.13) holds with probability greater than $1-\delta / 2$ for any $g_{n} \in \mathcal{G}_{a}$ (by properly tuning 
the probability), with the constant $C^{\prime}$ therein only depending on $C, M, c_{m}$. By going through the proof of (S.14) and (S.15), it can be shown that for $n \geq N$ and $g_{n} \in \mathcal{G}_{a}$, with probability larger than $1-\delta,\left\|\widehat{g}_{n, \lambda}-g_{n 0}-S_{n, \lambda}\left(g_{n 0}\right)\right\| \leq \widetilde{C} a_{n}$, where the constant $\widetilde{C}$ and $N$ are unrelated to $g_{n} \in \mathcal{G}_{a}$. Using (S.49) and by exactly the same proof of Theorem 5.3, it can be shown that $-2 n \cdot P L R T^{\prime}$ follows the same asymptotic normal distribution under $H_{1 n}: g=g_{n 0}$ as in Theorem 5.3, uniformly for $g_{n} \in \mathcal{G}_{a}$.

For notational simplicity, denote $R_{i}=\ell\left(Y_{i} ; g_{0}\left(Z_{i}\right)\right)-\ell\left(Y_{i} ; g_{n 0}\left(Z_{i}\right)\right)$ for $i=1, \ldots, n$. Secondly, we have

$$
E\left\{\left|\sum_{i=1}^{n}\left[R_{i}-E\left(R_{i}\right)\right]\right|^{2}\right\} \leq n E\left\{R_{i}^{2}\right\}=n E\left\{\left|\epsilon_{i} g_{n}\left(Z_{i}\right)+g_{n}\left(Z_{i}\right)^{2}\right|^{2}\right\}=O\left(n\left\|g_{n}\right\|^{2}+n\left\|g_{n}\right\|^{4}\right) .
$$

Therefore, uniformly over $g_{n} \in \mathcal{G}_{a}, n\left(\ell_{n, \lambda}\left(g_{0}\right)-\ell_{n, \lambda}\left(g_{n 0}\right)-E\left\{\ell_{n, \lambda}\left(g_{0}\right)-\ell_{n, \lambda}\left(g_{n 0}\right)\right\}\right)=O_{P}\left(n^{1 / 2}\left\|g_{n}\right\|+\right.$ $\left.n^{1 / 2}\left\|g_{n}\right\|^{2}\right)$. On the other hand, $E\left\{D S_{n, \lambda}\left(g_{n 0}\right) g_{n} g_{n}\right\}=-E\left\{\left|g_{n}(Z)\right|^{2}\right\}-\lambda J\left(g_{n}, g_{n}\right)=-\left\|g_{n}\right\|^{2}$. Therefore,

$E\left\{\ell_{n, \lambda}\left(g_{0}\right)-\ell_{n, \lambda}\left(g_{n 0}\right)\right\}=E\left\{S_{n, \lambda}\left(g_{n 0}\right)\left(-g_{n}\right)+(1 / 2) D S_{n, \lambda}\left(g_{n 0}\right) g_{n} g_{n}\right\}=\lambda J\left(g_{n 0}, g_{n}\right)-\left\|g_{n}\right\|^{2} / 2$.

Since $\left|J\left(g_{n 0}, g_{n}\right)\right| \leq\left|J\left(g_{0}, g_{n}\right)\right|+J\left(g_{n}, g_{n}\right) \leq J\left(g_{0}, g_{0}\right)^{1 / 2} \zeta^{1 / 2}+\zeta$, we get that $2 n\left(\ell_{n, \lambda}\left(g_{0}\right)-\right.$ $\left.\ell_{n, \lambda}\left(g_{n 0}\right)\right)=-n\left\|g_{n}\right\|^{2}+O_{P}\left(n \lambda+n^{1 / 2}\left\|g_{n}\right\|+n^{1 / 2}\left\|g_{n}\right\|^{2}\right)$ uniformly for $g_{n} \in \mathcal{G}_{a}$. This completes the proof.

S.17. Minimax rate of the PLRT test in general modeling framework. In this section, we remark that PLRT achieves the optimal minimax rate of hypothesis testing specified in [23] in a more general modeling framework. The proofs are similar to those of Theorem 5.4 but require a deeper technical tool, i.e., the mapping principle, which builds equivalence between the eigenvalues obtained under null and contiguous alternatives. Write the local alternative as $H_{1 n}: g=g_{n 0}$, where $g_{n 0}=g_{0}+g_{n}, g_{0} \in \mathcal{H}$ and $g_{n}$ belongs to some alternative value set $\mathcal{G}_{a}$ below.

TheOREM S.2. Let $m>(3+\sqrt{5}) / 4 \approx 1.309$ and $h \asymp n^{-d}$ for $\frac{1}{2 m+1} \leq d<\frac{2 m}{8 m-1}$. Let Assumption $A .1$ (a) hold for constants $C_{0}, C_{1}$, a compact interval $\mathcal{I}_{0}$ and an open interval $\mathcal{I}$ with $\mathcal{I}_{0} \subset \mathcal{I}$. Assume that there is a constant $C_{2}>0$ such that $1 / C_{2} \leq-\ddot{\ell}_{a}(Y ; a) \leq C_{2}$ holds for any $a \in \mathcal{I}$. The value of $2 g_{0}$ belongs to $\mathcal{I}_{0}$. Consider the alternative value set

$$
\mathcal{G}_{a}=\left\{g \in H^{m}(\mathbb{I}) \mid 2 g(z) \in \mathcal{I}_{0} \text { for any } z \in \mathbb{I},\|g\|_{\text {sup }} \leq \zeta, J(g, g) \leq M\right\}
$$

where $\zeta=1 /\left(2 C_{0} C_{1} C_{2}\right)$ and $M$ is a positive constant. Suppose, under $H_{1 n}: g=g_{n 0}$ for $g_{n} \in \mathcal{G}_{a}$, Assumptions $A .1$ (c) and A.2 hold (with $g_{0}$ therein replaced by $\left.g_{n 0}\right), E\left\{\epsilon_{n 0}^{4} \mid Z\right\} \leq C$, a.s., for some constant $C>0$ with $\epsilon_{n 0}=\dot{\ell}_{a}\left(Y ; g_{n 0}(Z)\right)$, and $\left\|\widehat{g}_{n, \lambda}-g_{n 0}\right\|=O_{P}\left(r_{n}\right)$ holds under $H_{1 n}: g=g_{n 0}$ 
uniformly over $g_{n} \in \mathcal{G}_{a}$. Then for any $\delta \in(0,1)$, there exist positive constants $C^{\prime}$ and $N$ such that

$$
\inf _{n \geq N} \inf _{\substack{g_{n} \in \mathcal{G}_{a} \\\left\|g_{n}\right\| \geq C^{\prime} \eta_{n}}} P\left(\text { reject } H_{0}^{\text {global }} \mid H_{1 n} \text { is true }\right) \geq 1-\delta,
$$

where $\eta_{n} \geq \sqrt{h^{2 m}+\left(n h^{1 / 2}\right)^{-1}}$. The minimal lower bound of $\eta_{n}$, i.e., $n^{-2 m /(4 m+1)}$, is achieved when $h=h^{* *} \equiv n^{-2 /(4 m+1)}$.

Proof of Theorem S.2. First of all, by direct calculations, one can verify by $\frac{1}{2 m+1} \leq d<$ $\frac{2 m}{8 m-1}$ and $m>\frac{3+\sqrt{5}}{4}$ that $h \asymp n^{-d}$ satisfies the conditions in Theorem 5.3. Throughout, we only consider $g_{n 0}=g_{0}+g_{n}$ for $g_{n} \in \mathcal{G}_{a}$.

Next we prove our theorem. We write

$$
-2 n \cdot P L R T_{n, \lambda}=-2 n\left(\ell_{n, \lambda}\left(g_{0}\right)-\ell_{n, \lambda}\left(g_{n 0}\right)\right)-2 n\left(\ell_{n, \lambda}\left(g_{n 0}\right)-\ell_{n, \lambda}\left(\widehat{g}_{n, \lambda}\right)\right) .
$$

The proof proceeds in two parts. We first note that $-2 n \cdot P L R T^{\prime} \equiv-2 n\left(\ell_{n, \lambda}\left(g_{n 0}\right)-\ell_{n, \lambda}\left(\widehat{g}_{n, \lambda}\right)\right)$ is actually the PLRT test for testing $H_{1 n}$ against $H_{1}^{\text {global }}$. Under $H_{1 n},-2 n \cdot P L R T^{\prime}$ has the same asymptotic distribution as described in Theorem 5.3, but uniformly for all $g_{n} \in \mathcal{G}_{a}$. That is to say, $\left(2 u_{n 0}\right)^{-1 / 2}\left(-2 n \cdot P L R T_{n, \lambda}^{\prime}-n\left\|W_{\lambda} g_{n 0}\right\|^{2}-h^{-1} \sigma_{K n 0}^{2}\right)=O_{P}(1)$ uniformly for $g_{n 0}=g_{0}+g_{n}$ with $g_{n} \in \mathcal{G}_{a}$, where $u_{n 0}=h^{-1} \sigma_{K n 0}^{4} / \rho_{K n 0}^{2}$ under $g=g_{n 0}$ and $\sigma_{K n 0}^{2}, \rho_{K n 0}^{2}$ are given in (5.14) with eigenvalues therein derived under $g=g_{n 0}$. Denote $u_{n}=h^{-1} \sigma_{K}^{4} / \rho_{K}^{2}$ under $g=g_{0}$ with $\sigma_{K}^{2}, \rho_{K}^{2}$ given in (5.14). Let $V_{g_{n 0}}$ and $V_{g_{0}}$ be the $V$ functionals defined as in Section 2.2 under $g=g_{n 0}$ and $g=g_{0}$ respectively. Then, for any $f \in \mathcal{H}$, by Assumption A.1 (a) and (b), we have

$$
\begin{aligned}
\left|V_{g_{n 0}}(f, f)-V_{g_{0}}(f, f)\right| & =\left|E\left\{\left[\ddot{\ell}_{a}\left(Y ; g_{n 0}(Z)\right)-\ddot{\ell}_{a}\left(Y ; g_{0}(Z)\right)\right]|f(Z)|^{2}\right\}\right| \\
& \leq E\left\{\sup _{a \in \mathcal{I}}\left|\ell_{a}^{\prime \prime \prime}(Y ; a)\right| \cdot\left|g_{n}(Z)\right| \cdot|f(Z)|^{2}\right\} \\
& \leq C_{0} C_{1} C_{2}\left\|g_{n}\right\|_{\sup } V_{g_{n 0}}(f, f)=\zeta_{0}\left\|g_{n}\right\|_{\sup } V_{g_{n 0}}(f, f),
\end{aligned}
$$

where $\zeta_{0}=C_{0} C_{1} C_{2}=1 /(2 \zeta)$ is a universal constant. Therefore, $\left(1-\zeta_{0}\left\|g_{n}\right\|_{\text {sup }}\right) V_{g_{n 0}}(f, f) \leq$ $V_{g_{0}}(f, f) \leq\left(1+\zeta_{0}\left\|g_{n}\right\|_{\text {sup }}\right) V_{g_{n 0}}(f, f)$. By the mapping principle (see Theorem 6.1 in [54]), the eigenvalues induced by the functional pairs $\left(V_{g_{n 0}}, J\right)$ and $\left(V_{g_{0}}, J\right)$ are thus equivalent in the sense that $\left(1-\zeta_{0}\left\|g_{n}\right\|_{\text {sup }}\right) \gamma_{\nu}^{n 0} \leq \gamma_{\nu} \leq\left(1+\zeta_{0}\left\|g_{n}\right\|_{\text {sup }}\right) \gamma_{\nu}^{n 0}$ for any $\nu \in \mathbb{N}$, where $\gamma_{\nu}^{n 0}$ denotes the eigenvalue corresponding to $V_{g_{n 0}}$ and $\gamma_{\nu}$ is the eigenvalue corresponding to $V_{g_{0}}$. Therefore, uniformly for $g_{n 0}$,

$$
\sigma_{K n 0}^{2}-\sigma_{K}^{2}=\sum_{\nu} \frac{h \lambda\left(\gamma_{\nu}-\gamma_{\nu}^{n 0}\right)}{\left(1+\lambda \gamma_{\nu}^{n 0}\right)\left(1+\lambda \gamma_{\nu}\right)}=O\left(\left\|g_{n}\right\|_{\text {sup }}\right)=O\left(h^{-1 / 2}\left\|g_{n}\right\|\right)
$$

Secondly, we show that $-2 n\left(\ell_{n, \lambda}\left(g_{0}\right)-\ell_{n, \lambda}\left(g_{n 0}\right)\right) \geq n C^{\prime}\left\|g_{n}\right\|^{2}+O_{P}\left(n^{1 / 2}\left\|g_{n}\right\|+n \lambda\right)$, where $C^{\prime}$ 
is some positive constant unrelated to $f$. Then

$$
\begin{aligned}
& \left(2 u_{n}\right)^{-1 / 2}\left(-2 n r_{K} \cdot P L R T-u_{n}\right) \\
= & r_{K}\left(2 u_{n}\right)^{-1 / 2}\left(-2 n \cdot P L R T_{n, \lambda}^{\prime}-n\left\|W_{\lambda} g_{n 0}\right\|^{2}-h^{-1} \sigma_{K n 0}^{2}\right)+r_{K}\left(2 u_{n}\right)^{-1 / 2} n\left\|W_{\lambda} g_{n 0}\right\|^{2} \\
& -r_{K}\left(2 u_{n}\right)^{-1 / 2} \cdot 2 n\left(\ell_{n, \lambda}\left(g_{0}\right)-\ell_{n, \lambda}\left(g_{n 0}\right)\right)+r_{K}\left(2 u_{n}\right)^{-1 / 2} h^{-1}\left(\sigma_{K n 0}^{2}-\sigma_{K}^{2}\right) \\
\geq & O_{P}(1)+n C^{\prime} r_{K}\left(2 u_{n}\right)^{-1 / 2}\left\|g_{n}\right\|^{2}\left(1+O_{P}\left(n^{-1 / 2}\left\|g_{n}\right\|^{-1}+\lambda\left\|g_{n}\right\|^{-2}\right)\right)+O\left(h^{-1}\left\|g_{n}\right\|\right),
\end{aligned}
$$

where $O_{P}(\cdot)$ holds uniformly for $g_{n} \in \mathcal{G}_{a}$. Let $n^{-1 / 2}\left\|g_{n}\right\|^{-1} \leq 1 / C, \lambda\left\|g_{n}\right\|^{-2} \leq 1 / C, C h^{-1}\left\|g_{n}\right\| \leq$ $n h^{1 / 2}\left\|g_{n}\right\|^{2}$, and $\left\|g_{n}\right\|^{2} \geq C\left(n h^{1 / 2}\right)^{-1}$ for sufficiently large $C$, which implies that $\left|\frac{-2 n r_{K} \cdot P L R T-u_{n}}{\left(2 u_{n}\right)^{1 / 2}}\right| \geq$ $c_{\alpha}$ with large probability, where $c_{\alpha}$ is the cutoff value (based on $N(0,1)$ ) for rejecting $H_{0}^{\text {global }}$ at nominal level $\alpha$. This means we have to assume $\left\|g_{n}\right\|^{2} \geq C\left(\lambda+\left(n h^{1 / 2}\right)^{-1}\right)$ to achieve large power.

Next we complete the above two parts. Firstly, it can be established that the following "uniform" FBR holds, i.e., for any $\delta \in(0,1)$, there exist positive constants $\widetilde{C}$ and $N$ such that

$$
\inf _{n \geq N} \inf _{g_{n} \in \mathcal{G}_{a}} P_{g_{n 0}}\left(\left\|\widehat{g}_{n, \lambda}-g_{n 0}-S_{n, \lambda}\left(g_{n 0}\right)\right\| \leq \widetilde{C} a_{n}\right) \geq 1-\delta
$$

where $a_{n}$ is defined in (3.5), The proof of (S.52) follows by a careful reexamination of Theorem 3.4. Specifically, one can choose $C$ and $M$ (to be unrelated to $g_{n} \in \mathcal{G}_{a}$ ) to be large so that the event $B_{n 1} \cap B_{n 2}$, which is defined in the proof of Theorem 3.4, has probability greater than $1-\frac{\delta}{4}$. Then by going through exactly the same proof, it can be shown that when $n \geq N$ for some suitably selected $N$, for any $g_{n} \in \mathcal{G}_{a},(\mathrm{~S} .13)$ holds with probability greater than $1-\delta / 2$ (by properly tuning the probability), with the constant $C^{\prime}$ therein only depending on $C, M, c_{m}$. By going through the proofs of (S.14) and (S.15), it can be shown that for $n \geq N$ and $g_{n} \in \mathcal{G}_{a}$, with probability larger than $1-\delta,\left\|\widehat{g}_{n, \lambda}-g_{n 0}-S_{n, \lambda}\left(g_{n 0}\right)\right\| \leq \widetilde{C} a_{n}$, where the constant $\widetilde{C}$ and $N$ are unrelated to $g_{n} \in \mathcal{G}_{a}$. Using (S.52) and by exactly the same proof of Theorem 5.3, it can be shown that $-2 n \cdot P L R T^{\prime}$ follows the same asymptotic normal distribution under $H_{1 n}: g=g_{n 0}$ as in Theorem 5.3, uniformly for $g_{n} \in \mathcal{G}_{a}$.

For simplicity, denote $R_{i}=\ell\left(Y_{i} ; g_{0}\left(Z_{i}\right)\right)-\ell\left(Y_{i} ; g_{n 0}\left(Z_{i}\right)\right)$ for $i=1, \ldots, n$. Then

$$
E\left\{\left|\sum_{i=1}^{n}\left[R_{i}-E\left(R_{i}\right)\right]\right|^{2}\right\} \leq n E\left\{R_{i}^{2}\right\}=n E\left\{\left|-\epsilon_{i} g_{n}\left(Z_{i}\right)+\ddot{\ell}_{a}\left(Y_{i} ; g_{n 0}^{*}\left(Z_{i}\right)\right) g_{n}\left(Z_{i}\right)^{2}\right|^{2}\right\}
$$

where $g_{n 0}^{*}(z)=g_{0}(z)+t^{*} g_{n}(z)$ for $t^{*} \in(0,1)$, implying $g_{n 0}^{*}(z) \in \mathcal{I}_{0}$ for any $z$. By Assumption A.1, we get that (S.53) is uniformly $O\left(n\left\|g_{n}\right\|^{2}\right)$ over $g_{n} \in \mathcal{G}_{a}$. Therefore, uniformly over $g_{n} \in \mathcal{G}_{a}$, $n\left(\ell_{n, \lambda}\left(g_{0}\right)-\ell_{n, \lambda}\left(g_{n 0}\right)-E\left\{\ell_{n, \lambda}\left(g_{0}\right)-\ell_{n, \lambda}\left(g_{n 0}\right)\right\}\right)=O_{P}\left(n^{1 / 2}\left\|g_{n}\right\|\right)$.

On the other hand, by $\sup _{a \in \mathcal{I}} \ddot{\ell}_{a}(Y ; a)<0$, we can find $C^{\prime}>0$ (unrelated to $g_{n} \in \mathcal{G}_{a}$ ) such that $E\left\{D S_{n, \lambda}\left(g_{n 0}^{*}\right) g_{n} g_{n}\right\}=E\left\{\ddot{\ell}_{a}\left(Y ; g_{n 0}^{*}(Z)\right)\left|g_{n}(Z)\right|^{2}\right\}-\lambda J\left(g_{n}, g_{n}\right) \leq-C^{\prime}\left\|g_{n}\right\|^{2} / 2$. Therefore,

$$
\begin{aligned}
E\left\{\ell_{n, \lambda}\left(g_{0}\right)-\ell_{n, \lambda}\left(g_{n 0}\right)\right\} & =E\left\{S_{n, \lambda}\left(g_{n 0}\right)\left(-g_{n}\right)+(1 / 2) D S_{n, \lambda}\left(g_{n 0}^{*}\right) g_{n} g_{n}\right\} \\
& \leq \lambda J\left(g_{n 0}, g_{n}\right)-C^{\prime}\left\|g_{n}\right\|^{2} / 2=O(\lambda)-C^{\prime}\left\|g_{n}\right\|^{2} / 2,
\end{aligned}
$$


where the last equality holds by $J\left(g_{n}, g_{n}\right) \leq M$ and $\left|J\left(g_{n 0}, g_{n}\right)\right| \leq\left|J\left(g_{0}, g_{n}\right)\right|+J\left(g_{n}, g_{n}\right) \leq$ $J\left(g_{0}, g_{0}\right)^{1 / 2} M^{1 / 2}+M$. Consequently, $2 n\left(\ell_{n, \lambda}\left(g_{0}\right)-\ell_{n, \lambda}\left(g_{n 0}\right)\right) \leq-n C^{\prime}\left\|g_{n}\right\|^{2}+O_{P}\left(n \lambda+n^{1 / 2}\left\|g_{n}\right\|\right)$. This completes the proof.

S.18. Proof of Lemma 6.1. We need the following two inequalities to establishing (6.3):

$$
\int_{0}^{\infty} \frac{1}{\left(1+x^{2 m}\right)^{l}} d x=\sum_{k=0}^{\infty} \int_{2 \pi h^{\dagger} k}^{2 \pi h^{\dagger}(k+1)} \frac{1}{\left(1+x^{2 m}\right)^{l}} d x \leq \sum_{k=0}^{\infty} \frac{2 \pi h^{\dagger}}{\left(1+\left(2 \pi h^{\dagger} k\right)^{2 m}\right)^{l}}
$$

and by a similar argument, $\int_{0}^{\infty} \frac{1}{\left(1+x^{2 m}\right)^{l}} d x \geq \sum_{k=1}^{\infty} \frac{2 \pi h^{\dagger}}{\left(1+\left(2 \pi h^{\dagger} k\right)^{2 m}\right)^{l}}$. This completes the proof.

University of Notre Dame

Notre Dame, IN 46545

EMAIL: ZUOFENGSHANG@GMAIL.COM
Department of Statistics Purdue University 250 N. University Street West Lafayette, IN 47906 EMAIL: CHENGG@STAT.PURduE.EdU 

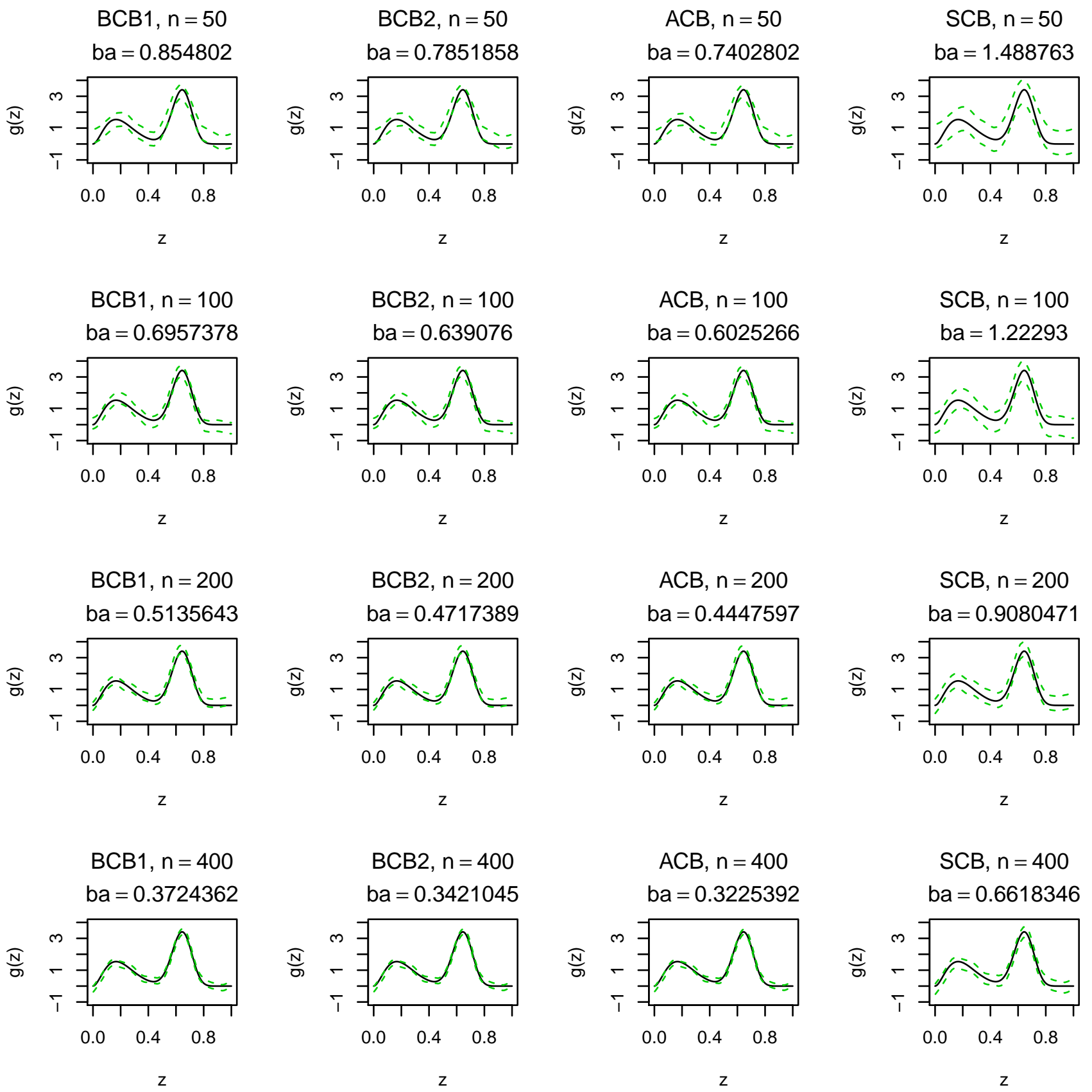

FIG 3. $95 \%$ pointwise and simultaneous confidence bands for periodic $g_{0}(z)=0.6 \beta_{30,17}(z)+0.4 \beta_{3,11}(z)$ in Case (I) of Example 6.1. The upper and lower bands are indicated by green curves, while the central black curve represents the true function. The numerical band area is denoted as "ba". 

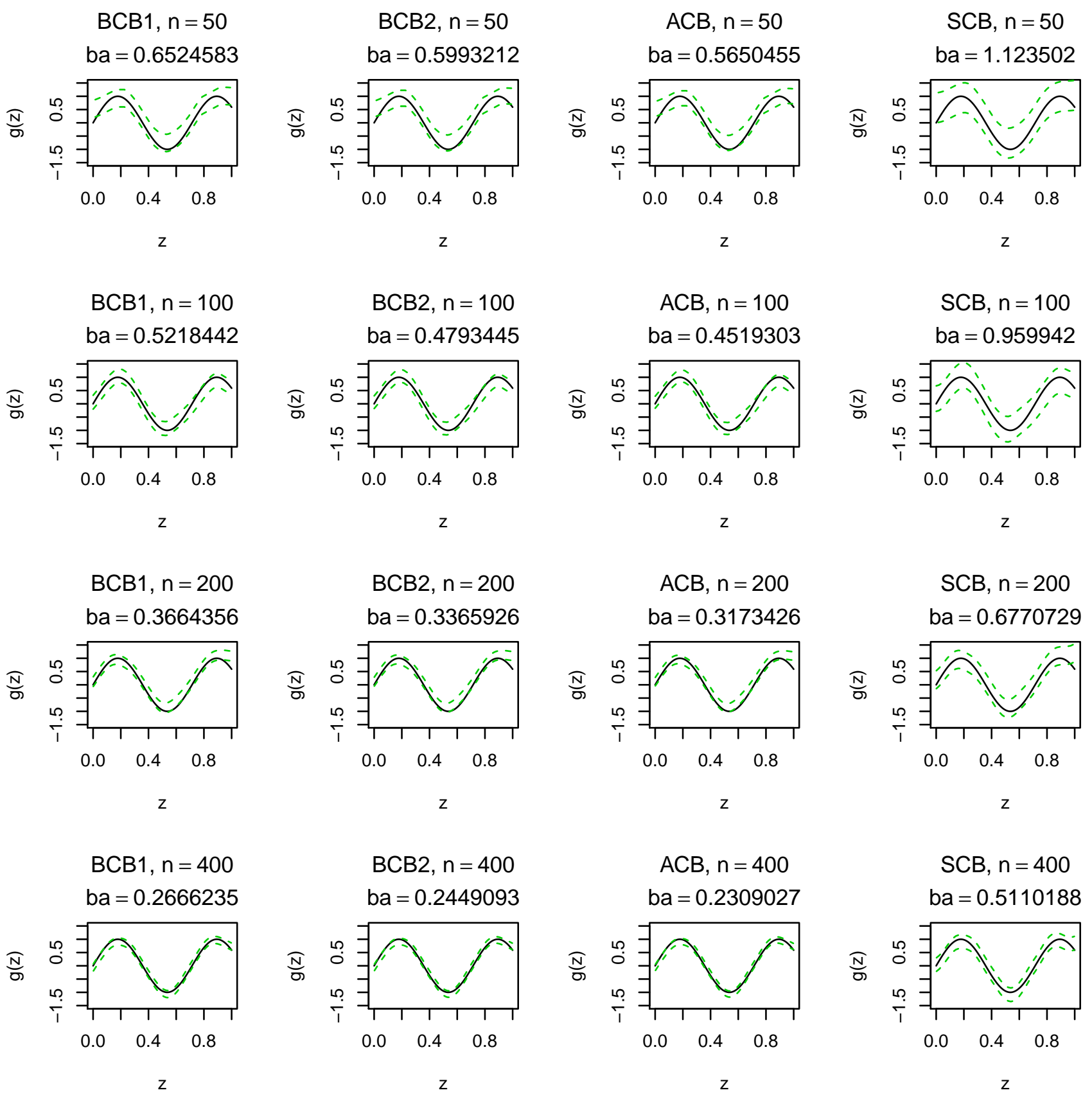

FIG 4. 95\% pointwise and simultaneous confidence bands for nonperiodic $g_{0}(z)=\sin (2.8 \pi z)$ in Case (II) of Example 6.1. The upper and lower bands are indicated by green curves, while the central black curve represents the true function. The numerical band area is denoted as "ba". 